\title{
On the Characterization of Relativistic Quantum Field Theories in Terms of Finitely Many Vacuum Expectation Values. I
}

\author{
Erwin Brüning \\ Fakultät für Physik der Universität, D-4800 Bielefeld, Federal Republic of Germany
}

\begin{abstract}
A characterization of monotone continuous linear functionals on tensoralgebras which arise in QFT is derived and some consequences are investigated. Then we look for necessary and sufficient conditions on a set
\end{abstract}

$$
T_{(N)}=\left\{1, T_{1}, T_{2}, \ldots, T_{N}\right\} \quad T_{n} \in E_{n}^{\prime}
$$

of " $n$-point-functionals", which guarantee the existence of at least one monotone continuous linear functional

$$
S=\left\{1, S_{1}, S_{2}, \ldots\right\} \quad \text { on } \quad \underline{E}=\bigoplus_{n=0}^{\infty} E_{n}, \quad E_{n}=E_{1} \tilde{\otimes}_{\pi} E_{1} \tilde{\otimes}_{\pi} \ldots \tilde{\otimes}_{\pi} E_{1},
$$

$E_{1}$ a special nuclear space, such that $S \uparrow \bigoplus_{n=0}^{N} E_{n}=T_{(N)}$, with special attention to QFT. A first application is a characterization of all monotone continuous linear extensions in the case $N=2$. The notion of minimal extensions is introduced. Its relevance is discussed. Necessary and sufficient conditions on $T_{(2 N)}$ for the existence of minimal extensions are presented. Some properties of minimal extensions are derived. In the simplest case $E \cong \mathbb{C}$ the concept of minimal extensions allows to answer the extension problem completely for arbitrary $N \in \mathbb{N}$. For the case of general $E=E_{1}$ and $N=2$ it is shown that the known examples of monotone continuous linear extensions are minimal extensions or a special generalization of it.

\section{Introduction, Notation}

Up to now the main concern in axiomatic QFT has been that of the linear program $[1,4,8 j$. The nonlinear constraints of QFT (positivity condition and uniqueness of the vacuum) have been treated much less $[15,17,19]$. But nevertheless these nonlinear constraints are as important as the linear constraints are. One step of incorporating the positivity condition into QFT is the program of Bros and Lasalle [20]. It relies on additional technical assumptions. 
It is well known $[12,13,4]$ that an axiomatic QFT in the sense of Wightman can be characterized in terms of some monotone continuous linear functional

$$
\mathscr{W}=\left\{1, \mathscr{W}_{1}, \mathscr{W}_{2}, \mathscr{W}_{3}, \ldots\right\}
$$

on the Borchers-algebra $\mathscr{S}=\bigoplus_{n=0}^{\infty} \mathscr{S}\left(\mathbb{R}^{4 n}\right)$.

This is a characterization of a QFT in terms of infinitely many vacuum expectation values $\mathscr{W}_{n}, n=1,2, \ldots$. Monotonicity is a restriction which connects the set of all $n$-point-functionals of a given theory at the same time and makes it thus hard to analyze.

We will try to do a step in the analysis of the positivity condition in QFT by characterizing the monotone continuous linear functionals on tensoralgebras such as the Borchers-algebra but forgetting for the moment the linear constraints and the uniqueness of the vacuum (Section 1 ).

As we think it to be adequate to use eventually other testfunction-spaces as $\mathscr{S}=\mathscr{S}\left(\mathbb{R}^{4}\right)$ or $\mathscr{D}=\mathscr{D}\left(\mathbb{R}^{4}\right)$, for instance Gelfand-spaces, in particular Jaffe-spaces $[1,2,10]$ we will formulate our investigations in terms of some abstract complete HLCTVS (Hausdorff locally convex topological vector space) $E=E_{1}$ over the field $\mathbb{C}$ of complex numbers.

Denote by $E_{n}$ the completion of the $n$-fold tensorproduct $E^{\otimes n}=E \otimes_{\pi} E \otimes_{\pi} \ldots \otimes_{\pi} E$ with respect to the projective topology $\tau_{n}$, induced by the given topology $\tau_{1}$ on $E . \tau_{n}$ is again a Hausdorff locally convex topology. Equip $E=\bigoplus_{n=0}^{\infty} E_{n}, E_{0}=\mathbb{C}$ with the topology $\tau$ of the locally convex direct sum with respect to the topologies $\tau_{n}$ and with the product

$$
\begin{aligned}
(\underline{a} \cdot \underline{b})_{n} & =\sum_{\nu+\mu=n} a_{v} \otimes b_{\mu}, \quad n=0,1,2, \ldots \\
\underline{a} & =\left\{a_{0}, a_{1}, \ldots, a_{N(a)}, 0,0, \ldots\right\} \in \underline{E}
\end{aligned}
$$

Then $\underline{E}$ is an associative algebra with unit $1=\{1,0,0, \ldots\}$. Call it the complete tensoralgebra of $E$. By [7, Theorem II.6.2] $E$ is again a complete HLCTVS. The tensoralgebra of $E$ is

$$
\mathscr{T}(E)=\sum_{n=0}^{\infty}\left(E^{\otimes n}, \tau_{n}\right)
$$

it gives by completion just $\underline{E}$.

We assume that there is a continuous involution $*$ on $E$; it has a unique extension, which is a continuous involution on $\underline{E}$, and satisfies

$$
1^{*}=1 \quad(\underline{a} \cdot \underline{b})^{*}=\underline{b}^{*} \cdot \underline{a}^{*} .
$$

As a positive wedge $\underline{E}_{+}$in $\underline{E}$ we take the set

$$
\underline{E}_{+}=\left\{\sum_{j=1}^{N} \underline{a}_{j}^{*} \cdot \underline{a}_{j} \mid \underline{a}_{j} \in \underline{E}, N \in \mathbb{N}\right\},
$$

$E$ is then generated by this wedge:

$$
\underline{E}=\underline{E}_{+}-\underline{E}_{+}+i\left(\underline{E}_{+}-\underline{E}_{+}\right)
$$


This follows from the fact that the algebra $E$ has a unit, using a polarization identity. The space of continuous linear functionals on $\underline{E}$ is denoted by $\underline{E}^{\prime}$ and is given by $\underline{E}^{\prime}=\prod_{n=0}^{\infty} E_{n}^{\prime}$, e.g. $\underline{E}^{\prime}$ is the topological product of the dual spaces $E_{n}^{\prime}$ of the spaces $E_{n}[7 ;$ IV.4.3.1].

We want to investigate the set

$$
\underline{E}_{+}^{\prime}=\left\{T \in \underline{E}^{\prime} \mid T \uparrow \underline{E}_{+} \geqq 0\right\}
$$

of monotone (e.g. non negative) continuous linear (m.c.l.) functionals on $\underline{E}$ and in particular the structure of its elements. To do this effectively (in the sense of possible application to QFT) we clearly have to assume some additional structure of $E$. This will be done later.

For the moment we note some easy consequences of monotonicity: For $T \in \underline{E}_{+}^{\prime}$ we have a positive semi-definite sesquilinear form on $\underline{E}$ :

$$
(\underline{a}, \underline{b}) \mapsto T\left(\underline{a}^{*} \cdot \underline{b}\right)
$$

and thus by Schwarz-inequality

$$
\left|T\left(\underline{a}^{*} \cdot \underline{b}\right)\right| \leqq T\left(\underline{a}^{*} \cdot \underline{a}\right)^{1 / 2} T\left(\underline{b}^{*} \cdot \underline{b}\right)^{1 / 2}
$$

in particular

$$
|T(\underline{a})|^{2} \leqq T(\underline{1}) T\left(\underline{a}^{*} \cdot \underline{a}\right)
$$

and thus

$$
T(\underline{1})=0 \Rightarrow T=0 .
$$

Therefore $\underline{E}_{+}^{\prime}$ is a convex cone with a basis

$$
\underline{E}_{+, 1}^{\prime}=\left\{T \in \underline{E}_{+}^{\prime} \mid T(\underline{1})=1\right\} .
$$

A further immediate consequence of (2a) is the following: If $T \in \underline{E}_{+, 1}^{\prime}$ then

$$
(\underline{a}, \underline{b}) \mapsto B_{T}(\underline{a}, \underline{b})=T\left(\underline{a}^{*} \cdot \underline{b}\right)-T\left(\underline{a}^{*}\right) T(\underline{b})
$$

is again a positive semidefinite sesquilinear form on $\underline{E}$. This gives by well known criteria an infinite set of inequalities connecting all $T_{n}, n \in \mathbb{N}$, of $T=\left\{1, T_{1}, T_{2}, \ldots\right\} \in \underline{E}_{+, 1}^{\prime} ;$ some of the simplest one's are

$$
\left|\left(T_{n+m}-T_{n} \otimes T_{m}\right)\left(a_{n}^{*} \otimes b_{m}\right)\right|^{2} \leqq T_{n n}\left(a_{n}^{*} \otimes a_{n}\right) T_{m m}\left(b_{m}^{*} \otimes b_{m}\right)
$$

for all $a_{n} \in E_{n}$, all $b_{m} \in E_{m}, n, m=1,2,3, \ldots$; where we defined $\left(T_{n} \otimes T_{m}\right)\left(a_{n} \otimes b_{m}\right)$ $=T_{n}\left(a_{n}\right) T_{m}\left(b_{m}\right)$ and $T_{n n}=T_{2 n}-T_{n} \otimes T_{n}$. Our further assumptions are mainly motivated by the application in QFT, which we have in mind.

Assumptions.

(a) $E$ (all $\left.E_{n}\right)$ is (are) barreled [7],

(b) $E$ is a nuclear space $[5,7]$.

These assumptions are known to be true for the proposed testfunction spaces of QFT: $\mathscr{S}\left(\mathbb{R}^{4}\right), \mathscr{D}\left(\mathbb{R}^{4}\right)$ and some Gelfand(-Jaffe-)spaces $\mathscr{S}_{g}\left(\mathbb{R}^{4}\right)$. This has been stated 
for $\mathscr{S}\left(\mathbb{R}^{4}\right)$ in [13] and can be done similarly for the others using standard results in the theory of LCTVS $[2,6,7,9,10]$. If $E$ is nuclear, then all spaces $E_{n}$ are nuclear [7,III.7.5] and thus $\underline{E}=\bigoplus_{n=0}^{\infty} E_{n}$ is nuclear [7,III.7.4].

The nuclear-theorem $[5,2,7]$ tells us in which way each $T_{n} \in E_{n}^{\prime}$ is constructed out of certain equicontinuous sequences of elements in $E^{\prime}$ :

$$
T_{n} \in E_{n}^{\prime} \quad \text { iff } \quad T_{n}\left(x_{1} \otimes \ldots \otimes x_{n}\right)=\sum_{j_{1}, \ldots, j_{n}=1}^{\infty} \lambda_{j_{1}}^{(1)} \ldots \lambda_{j_{n}}^{(n)} c_{n}\left(j_{1}, \ldots, j_{n}\right) t_{j_{1}}^{(1)}\left(x_{1}\right) \ldots t_{j_{n}}^{(n)}\left(x_{n}\right)
$$

with the following specifications
(i) $\sum_{j=1}^{\infty}\left|\lambda_{j}^{(v)}\right|<\infty \quad v=1, \ldots, n$
(ii) $\left|c_{n}\left(j_{1}, \ldots, j_{n}\right)\right| \leqq 1$
(iii) $\left|t_{j_{v}}^{(v)}(x)\right| \leqq p_{v}(x)$
$j_{v} \in \mathbb{N}, \quad v=1, \ldots, n$

$p_{v}$ some continuous seminorm on $E, v=1, \ldots, n$.

We call the representation (6) a nuclear representation of $T_{n} \in E_{n}^{\prime}$.

In Section 1 we show that for $T \in E_{+, 1}^{\prime}$ these nuclear representations of the various $T_{n}$ fit together in such a nice way that characterizes monotonicity conveniently. The tool we use is that of representing unbounded symmetric operators by infinite matrices. By [18] it is known that this representation is not a very helpful method for the investigation of unbounded operators, because there is no uniqueness relation, and they have complicated transformation properties under unitary matrices. But as we are interested only in representations of the functionals $T \in \underline{E}_{+, 1}^{\prime}$ and not in the associated representation operators $A_{T}(\underline{a}), \underline{a} \in \underline{E}$ in the $G N S$-construction, this nonuniqueness does not produce any additional difficulties.

We use the nuclear representation for $T \in \underline{E}_{+, 1}^{\prime}$ to investigate some structural aspects of $\underline{E}_{+, 1}^{\prime}$, thus getting a slight generalization of some results of [13] and some convenient recursion formulae for $T \in \underline{E}_{+, 1}^{\prime}$.

Section 2 treats the following kind of extension problem:

Given

$$
T_{(N)}=\left\{1, T_{1}, \ldots, T_{N}\right\} \quad T_{n} \in E_{n}^{\prime}
$$

which conditions guarantee the existence of some $T \in \underline{E}_{+, 1}^{\prime}$ such that

$$
T \uparrow \bigoplus_{n=0}^{N} E_{n}=T_{(N)} \text {. }
$$

Clearly a practicable answer to this problem would be of considerable importance in QFT. It would be an essential step in proving the existence of nontrivial QFT's. In Part II the case of a continuous linear functional $T_{(2 N)}$ which determines exactly one relativistic QFT is isolated. To answer this problem in the context of QFT in the general case is much harder because the linear constraints of QFT have to be taken into account in addition to monotonicity. 
We propose a strategy to attack this kind of extension problems (Section 2). This helps

(i) to understand why the extension problem for $N \leqq 2$ is trivial but not for $N>2$ (Section 3 ),

(ii) to characterize the set of all extensions for $N \leqq 2$ (Section 3),

(iii) It leads to the concept of minimal extensions (Section 2).

A discussion on the relevance of this concept follows and necessary and sufficient conditions on $T_{(2 N)}$ for the existence of minimal extensions are derived (Section 2). In Section 4 the concept of minimal extensions is applied to the simplest case $E \cong \mathbb{C}$. This allows to answer the above extension problem completely for arbitrary $N$.

\section{On the Structure of $T \in \underline{E}_{+, 1}^{\prime}$}

The aim of this section is to give a complete characterization of a given m.c.l. functional $T$ on $E$ in terms of its nuclear representations. By construction $\underline{a} \mapsto \underline{c}^{*} \cdot \underline{a} \cdot \underline{b}$ is a continuous linear map $\underline{E} \rightarrow \underline{E}$ for each fixed $\underline{c}, \underline{b} \in \underline{E}$; thus given $T \in \underline{E}^{\prime}$ and $\underline{b}, \underline{c} \in \underline{E} \underline{a} \mapsto T_{\underline{b}, \underline{c}}(\underline{a})=T\left(\underline{c}^{*} \cdot \underline{a} \cdot \underline{b}\right)$ is again a continuous linear functional on $\underline{E}$. For m.c.l. functionals on a barreled space $\underline{E}$ one can prove much more [16].

Lemma 1.1. Given $T \in \underline{E}_{+, 1}^{\prime}$ define

$$
q_{T}(\underline{a})=T\left(\underline{a}^{*} \cdot \underline{a}\right)^{1 / 2} .
$$

Assume $\underline{E}$ to be barreled. Then for each fixed $\underline{b} \in \underline{E}$

$$
\underline{a} \mapsto q_{T}(\underline{a} \cdot \underline{b})
$$

defines a continuous seminorm on $\underline{E}$.

Proof. It is immediate that $q_{T}(\cdot \underline{b})$ defines a seminorm on $\underline{E}$. To prove continuity we define for fixed $\underline{b} \in \underline{E}$ :

$$
\mathscr{T}_{b}=\left\{T_{\underline{b}, \underline{c}} \mid q_{T}(\underline{c}) \leqq 1\right\} .
$$

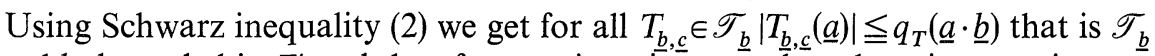
is weakly bounded in $E^{\prime}$ and therefore equicontinuous; thus there is a continuous seminorm $q_{\underline{b}}$ on $\underline{E}$ such that $\left|T_{\underline{b}, \underline{c}}(\underline{a})\right| \leqq q_{\underline{b}}(\underline{a})$ for all

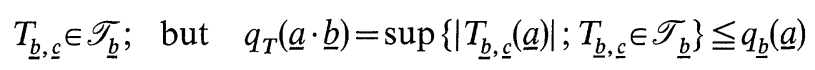

which proves the assertion.

In order to get a convenient formulation we have to introduce some more notation. Let us denote by $\mathfrak{M}^{(2)}$ the set of all square summable infinite matrices of complex numbers, e.g. $\left(a_{v \mu}\right)_{v, \mu \in \mathbb{N}} \in \mathfrak{M}^{(2)}$ iff $\sum_{v=1}^{\infty}\left|a_{v \mu}\right|^{2}<\infty, \sum_{\mu=1}^{\infty}\left|a_{v \mu}\right|^{2}<\infty$. Then define $\mathfrak{M}^{(2)}\left(E^{\prime}\right)$ to be the set of all *-homomorphisms $m$ from $E$ into $\mathfrak{M}^{(2)}$ such that for all $\mu \in \mathbb{N}$

$$
x \mapsto\left(\sum_{v=1}^{\infty}\left|m_{v \mu}(x)\right|^{2}\right)^{1 / 2}=q_{\mu}(x)
$$


is a continuous seminorm on $E . m$, being a *-homomorphism satisfies

$$
m_{v \mu}(x)=\overline{m_{\mu v}\left(x^{*}\right)}
$$

and thus

$$
\left(\sum_{\mu=1}^{\infty}\left|m_{v \mu}(x)\right|^{2}\right)^{1 / 2}=q_{\mu}\left(x^{*}\right) .
$$

Next we introduce the set $\mathfrak{M}_{\infty}^{(2)}\left(E^{\prime}\right)$ of all $m \in \mathfrak{M}^{(2)}\left(E^{\prime}\right)$ which have the following property

(a) $m(E)$ generates an associative *-algebra $\mathfrak{U}(m)$ in $\mathfrak{M}^{(2)}$

(b) $\underset{n \in \mathbb{N}}{\Lambda} x_{1} \otimes \ldots \otimes x_{n} \mapsto\left(\sum_{v=1}^{\infty}\left|\left(m\left(x_{1}\right) \ldots m\left(x_{n}\right)\right)_{v \mu}\right|^{2}\right)^{1 / 2}$

is a continuous seminorm on $E^{\otimes n}$, called $q_{\mu}^{(n)}$.

We think these matrices to act in some Hilbertspace $l^{2}$ of square summable sequences $\underline{z}$ of complex numbers $\left(z_{v}\right)_{v \in \mathbb{N}}$. Then the subspace $l_{e}^{2}$ of terminating sequences is always in the domain of all these matrices. Our interest in this set $\mathfrak{M}_{\infty}^{(2)}\left(E^{\prime}\right)$ results from its intimate connection with monotone continuous linear functionals on $\underline{E}$ :

Theorem 1.2. Let $\underline{E}$ be the complete tensoralgebra of a HLCTVS E, which has the properties (5). Then a continuous linear functional $T$ on $E$ is monotone if and only if there is a $m=m_{T} \in \mathfrak{M}_{\infty}^{(2)}\left(E^{\prime}\right)$ such that the restriction of $T$ to $\mathscr{T}(E)$ has the following representation in terms of $m$. For all $n \in \mathbb{N}$ and all $x_{j} \in E$ we have

$$
\begin{aligned}
T_{n}\left(x_{1} \otimes \ldots \otimes x_{n}\right) & =\left\langle\underline{e}_{0}, m\left(x_{1}\right) \ldots m\left(x_{n}\right) \underline{e}_{0}\right\rangle_{2} \\
& =\sum_{j_{1}, \ldots, j_{n-1}=0}^{\infty} m_{0 j_{1}}\left(x_{1}\right) m_{j_{1} j_{2}}\left(x_{2}\right) \ldots m_{j_{n-1} 0}\left(x_{n}\right) .
\end{aligned}
$$

These series have to be evaluated successively in arbitrary order; in each step we have absolute convergence.

The matrix $m=\left(m_{v \mu}\right)_{v \mu \in \mathbb{N}}$ is not uniquely determined by Equation (11). The most general such matrix is obtained from a given $m$ in terms of a unitary transformation $u$

$$
\hat{m}=u m u^{*}
$$

in $\mathfrak{M}_{\infty}^{(2)}\left(E^{\prime}\right)$ :

$$
T_{n}\left(x_{1} \otimes \ldots \otimes x_{n}\right)=\left\langle u \underline{e}_{0}, \hat{m}\left(x_{1}\right) \ldots \hat{m}\left(x_{n}\right) u \underline{e}_{0}\right\rangle_{2} .
$$

Remark. Dealing with infinite matrices the transformation (12) causes some difficulties. These transformations have to be understood in the sense of v. Neumann [18], which essentially means that $u^{*} \underline{e}_{n}$ is in the domain of $m(x)$ for all $n \in \mathbb{N}$ and all $x \in E$ sharpened by the requirement that the transformed matrix $\hat{m}$ has to be again in $\mathfrak{M}_{\infty}^{(2)}\left(E^{\prime}\right)$ and $u \underline{e}_{n}$ is in the domain of all $\hat{m}\left(x_{1}\right) \ldots \hat{m}\left(x_{n}\right), x_{j} \in E, n \in \mathbb{N}$. $\left\{\underline{e}_{n} ; n=0,1,2, \ldots\right\}$ denotes the standard basis of $l^{2}$.

This theorem is essentially known. Therefore we do not prove it but comment on it introducing by this way some notation which is used later. 
At first one realizes that each $T \in \underline{E}_{+, 1}^{\prime}$ has a canonical pre-Hilbertspace realization

$$
V_{T}=\left(\Phi_{T}(\underline{E}),\langle\cdot, \cdot\rangle_{T}\right)
$$

where $\Phi_{T}: \underline{E} \rightarrow \underline{E} / q_{T}^{-1}(0) \equiv \Phi_{T}(\underline{E})$ is the canonical quotient map onto the factorspace of $\underline{E}$ with respect to the closed subspace $q_{T}^{-1}(0)$,

$$
q_{T}(\underline{x})=T\left(\underline{x}^{*} \cdot \underline{x}\right)^{1 / 2} \quad \underline{x} \in \underline{E},
$$

and where the scalarproduct is defined by

$$
\left\langle\Phi_{T}(\underline{x}), \Phi_{T}(\underline{y})\right\rangle_{T}=T\left(\underline{x}^{*} \cdot \underline{y}\right) \quad \underline{x}, \underline{y} \in \underline{E} .
$$

The completion of $V_{T}$ gives a Hilbertspace $\mathscr{H}_{T}=\tilde{V}_{T}$ whose scalarproduct is also denoted by $\langle\cdot, \cdot\rangle_{T}$. The nuclearity of $\underline{E}$ implies the nuclearity of $\Phi_{T}$ and thus the separability of $\mathscr{H}_{T}[2,27.4 .3]$.

Then one sees that by Schwarz-inequality (2) $q_{T}^{-1}(0)$ is a left ideal in $\underline{E}$ so that a weakly continuous cyclic *-representation with cyclic vector $\Phi_{0}=\Phi_{T}(\underline{1})$

$$
\underline{A}_{T}: \underline{E} \rightarrow L\left(V_{T}, V_{T}\right)
$$

is welldefined by

$$
\underline{A}_{T}(\underline{x}) \Phi_{T}(\underline{y})=\Phi_{T}(\underline{x} \cdot \underline{y}) .
$$

As usual we denote by $L\left(V_{T}, V_{T}\right)$ the space of linear functions from $V_{T}$ into $V_{T}$. By assumption (5a) and L. 1.1 we get that $\underline{x} \mapsto\left\|\underline{A}_{T}(\underline{x}) \Phi_{T}(\underline{y})\right\|_{T}=q_{T}(\underline{x} \cdot \underline{y})$ is a continuous seminorm on $\underline{E}$ for each fixed $\underline{y} \in \underline{E}$; e.g. the representation $\underline{A}_{T}$ is strongly continuous.

By definition of $\underline{E}$ one realizes that the above representation $\underline{A}_{T}$ of $\underline{E}$ on $V_{T}$ is fixed in terms of a linear map (a 'field')

$$
\begin{aligned}
& A_{T}: E_{1} \rightarrow L\left(\mathscr{D}_{A_{T}}, \mathscr{D}_{A_{T}}\right) \\
& \mathscr{D}_{A_{T}}=\Phi_{T}(\mathscr{T}(E))=\Phi_{T}\left(\bigoplus_{n=0}^{\infty} E_{1}^{\otimes n}\right)=\bigcup_{n=1}^{\infty} \mathscr{D}_{n} \\
& \mathscr{D}_{1}=\mathbb{C} \Phi_{0}, \quad \mathscr{D}_{n+1}=\operatorname{lin}\left(\mathscr{D}_{n} \cup A_{T}\left(E_{1}\right) \mathscr{D}_{n}\right), \quad n=1,2, \ldots
\end{aligned}
$$

such that

$$
\begin{aligned}
& A_{T}(x)^{*}\left\lceil\mathscr{D}_{A_{T}}=A_{T}\left(x^{*}\right) \uparrow \mathscr{D}_{A_{T}} \text { for all } x \in E_{1}\right. \\
& p_{n}\left(x_{1} \otimes \ldots \otimes x_{n}\right)=\left\|A_{T}\left(x_{1}\right) \ldots A_{T}\left(x_{n}\right) \Phi_{0}\right\|_{T}
\end{aligned}
$$

is a continuous seminorm on $E_{1}^{\otimes n}, n=1,2, \ldots$.

Again $\mathscr{D}_{A_{T}} \subseteq \Phi_{T}(\underline{E})$ is dense in $\mathscr{H}_{T}$. By construction we have

$$
T_{n}\left(x_{1} \otimes \ldots \otimes x_{n}\right)=\left\langle\Phi_{0}, A_{T}\left(x_{1}\right) \ldots A_{T}\left(x_{n}\right) \Phi_{0}\right\rangle_{T} \quad \text { all } \quad x_{j} \in E_{1}, \quad n \in \mathbb{N}
$$

and we know that all $A_{T}\left(x_{1}\right) \ldots A_{T}\left(x_{n}\right)=\underline{A}_{T}\left(j_{n}\left(x_{1} \otimes \ldots \otimes x_{n}\right)\right)$ have an extension defined on $\Phi_{T}(E)\left(j_{n}: E_{n} \rightarrow \underline{E}\right.$ denotes the natural injection, $\left.n=0,1,2, \ldots\right)$. Therefore we may choose any orthonormal basis $\left\{\varphi_{j}\right\}_{j=0,1,2, \ldots \subseteq} \Phi_{T}(E)$ of $\mathscr{H}_{T}$ starting with 
$\varphi_{0}=\Phi_{0}$ and then define the matrix representation of $A_{T}(x)$ with respect to this basis, e.g.

$$
\begin{aligned}
m(x) & \equiv m_{T}(x)=\left(m_{v \mu}(x)\right) \quad v, \mu=0,1,2, \ldots \\
m_{v \mu}(x) & =\left\langle\varphi_{v}, A_{T}(x) \varphi_{\mu}\right\rangle_{T} .
\end{aligned}
$$

This $m$ then belongs to $\mathfrak{M}_{\infty}^{(2)}\left(E^{\prime}\right)$ and has the properties as claimed.

The converse that each $m \in \mathfrak{M}_{\infty}^{(2)}\left(E^{\prime}\right)$ and each cyclic vector $\underline{z} \in l^{2}$ for $m$ defines an element

$$
T=T_{m, \underline{z}} \in \underline{E}_{+, 1}^{\prime}
$$

according to

$$
T_{n}\left(x_{1} \otimes \ldots \otimes x_{n}\right)=\left\langle\underline{z}, m\left(x_{1}\right) \ldots m\left(x_{n}\right) \underline{z}\right\rangle_{2}
$$

is fairly obvious.

The transformation properties (12) express the possibility of choosing different orthonormal basis in $\Phi_{T}(\underline{E})$ for the definition of a matrix representation of $A_{T}$.

Theorem 1.2 says that each state (normalized continuous monotone linear functional) $T \in \underline{E}_{+, 1}^{\prime}$ is given in terms of a certain matrix $m \in \mathfrak{M}_{\infty}^{(2)}\left(E^{\prime}\right)$ with coefficients in $E^{\prime}$ and a certain vector $\underline{z}$ in $l^{2}$. Therefore we may rephrase Theorem 1.2 to say that each state $T$ on $\underline{E}$ is a matrix-state $T_{m, \underline{z}}$. Thus a detailed knowledge of $\mathfrak{M}_{\infty}^{(2)}\left(E^{\prime}\right)$ is equivalent to a detailed knowledge of $\underline{E}_{+, 1}^{\prime}$. In particular one would like to have a convenient characterization of the structure of elements in $\mathfrak{M}_{\infty}^{(2)}\left(E^{\prime}\right)$. This can be done for the simplest case (Section 4, Proposition 4.3). The result then is that each such matrix $m$ is equivalent to a Jacobi-matrix. But this is not known to hold in general. Therefore we discuss some vectorspaces of elements in $\mathfrak{M}_{\infty}^{(2)}\left(E^{\prime}\right)$ which are dense in a sense which we will specify in a moment.

First we introduce the vectorspace

$$
\begin{aligned}
& \mathfrak{M}^{(\infty)}\left(E^{\prime}\right) \\
& \quad=\left\{m=\left(m_{v \mu}\right)_{v, \mu \in \mathbb{N}} \mid m_{v \mu} \in E^{\prime} ; m(x)^{*}=m\left(x^{*}\right) ; m_{v \mu}=0, v>N(m) \text { or } \mu>N(m)\right\}
\end{aligned}
$$

of all finite dimensional matrices with coefficients in $E^{\prime}$, embedded in $\mathfrak{M}^{(2)}\left(E^{\prime}\right)$. Clearly for $m \in \mathfrak{M}^{(\infty)}\left(E^{\prime}\right)$ we have for any $\underline{z} \in l^{2}, n \in \mathbb{N}, x_{j} \in E$

$$
\left\|m\left(x_{1}\right) \ldots m\left(x_{n}\right) \underline{z}\right\|_{2} \leqq\left\|m\left(x_{1}\right)\right\| \ldots\left\|m\left(x_{n}\right)\right\|\|\underline{z}\|_{2}
$$

thus $\mathfrak{M}^{(\infty)}\left(E^{\prime}\right) \subseteq \mathfrak{M}_{\infty}^{(2)}\left(E^{\prime}\right)$ and each $\underline{z} \in l^{2},\|\underline{z}\|_{2}=1$ each $m \in \mathfrak{M}^{(\infty)}\left(E^{\prime}\right)$ give rise to a state $T_{m, \underline{z}}$ on $\underline{E}$. We call the set

$$
\left\{T_{m, \underline{z}} \mid m \in \mathfrak{M}^{(\infty)}\left(E^{\prime}\right), \underline{z} \in l^{2},\|\underline{z}\|_{2}=1\right\}
$$

the set of finite order matrix states on $\underline{E}$. Restricted to $\bigoplus_{n=0}^{\infty} E^{\otimes n}$ a matrix state of order $N$ is of the form

$$
T_{m, \underline{z} ; n}^{(N)}\left(x_{1} \otimes \ldots \otimes x_{n}\right)=\sum_{v, \mu, j_{1}, \ldots, j_{n-1}=0}^{N} \bar{z}_{v} m_{v j_{1}}\left(x_{1}\right) m_{j_{1} j_{2}}\left(x_{2}\right) \ldots m_{j_{n-1} \mu}\left(x_{n}\right) z_{\mu} .
$$


By Theorem 1.2 it is almost trivial that the set of all finite order matrix states is dense in the set $\underline{E}_{+, 1}^{\prime}$ of all states on $\underline{E}$, indeed we have

Corollary 1.3. Assume $\underline{E}$ to be as in Theorem 1.2. Then each state $T$ on $\underline{E}$ is the limit of a sequence of finite order matrix states $\left\{T^{(N)}\right\}_{N \in \mathbb{N}}$.

Proof. By Theorem 1.2 each $T \in \underline{E}_{+, 1}^{\prime}$ is a matrix state, that is there is a $m \in \mathfrak{M}_{\infty}^{(2)}\left(E^{\prime}\right)$ such that we have on $\mathscr{T}(E)$ :

$$
T_{n}\left(x_{1} \otimes \ldots \otimes x_{n}\right)=\left\langle\underline{e}_{0}, m\left(x_{1}\right) \ldots m\left(x_{n}\right) \underline{e}_{0}\right\rangle_{2} .
$$

Define $P_{n}$ to be the orthogonal projection onto the subspace of $l^{2}$ spanned by $\left\{\underline{e}_{0}, \underline{e}_{1}, \ldots, \underline{e}_{n}\right\}$. Then one proves that

$$
P_{N} m\left(x_{1}\right) P_{N} m\left(x_{2}\right) P_{N} \ldots P_{N} m\left(x_{n}\right) P_{N} \underline{e}_{0} \underset{N \rightarrow \infty}{\longrightarrow} m\left(x_{1}\right) m\left(x_{2}\right) \ldots m\left(x_{n}\right) \underline{e}_{0}
$$

in $l^{2}$ and thus

$$
T_{n}^{(N)}\left(x_{1} \otimes \ldots \otimes x_{n}\right)=\left\langle\underline{e}_{0}, P_{N} m\left(x_{1}\right) P_{N} \ldots P_{N} m\left(x_{n}\right) P_{N} \underline{e}_{0}\right\rangle_{2} \underset{N \rightarrow \infty}{\longrightarrow} T_{n}\left(x_{1} \otimes \ldots \otimes x_{n}\right) .
$$

Therefore the sequence $T^{(N)}=\left\{T_{n}^{(N)} ; n=0,1,2, \ldots\right\}$ of finite order matrix states converges weakly on $\mathscr{T}(E)$ to $T\left\lceil\mathscr{T}(E)\right.$ and we know $T^{(N)} \in \underline{E}_{+, 1}^{\prime}$ and $T \in \underline{E}_{+, 1}^{\prime}$.

Remark. The above result generalizes Theorem II. 3.8 of [13] where for $E=\mathscr{S}\left(\mathbb{R}^{4}\right)$ it is proven that each state on $\underline{E}=\mathscr{L}$ is the limit of a net of finite order matrix states.

For explicit constructions of elements in $\underline{E}_{+, 1}^{\prime}$ starting from elements in $\mathfrak{M}_{\infty}^{(2)}\left(E^{\prime}\right)$ the following vectorspace $D\left(E^{\prime}\right)$ in $\mathfrak{M}_{\infty}^{(2)}\left(E^{\prime}\right)$ seems to be more interesting than $\mathfrak{M}^{(\infty)}\left(E^{\prime}\right)$ : We define $D\left(E^{\prime}\right)$ to be the set of all $m$ in $\mathfrak{M}^{(2)}\left(E^{\prime}\right)$ such that for each $\nu \in \mathbb{N}$ there exists $N_{v} \in \mathbb{N}$ with

(i) $m_{v \mu}=0$ for all $\mu>N_{v}$

(ii) $N_{v+1} \leqq n_{0}+N_{v}$

e.g. $D\left(E^{\prime}\right)$ is the set of all infinite matrices whose coefficients are different from zero only near the diagonal $v=\mu$. Clearly $D\left(E^{\prime}\right)$ is a vectorspace contained in $\mathfrak{M}^{(2)}\left(E^{\prime}\right)$. Furthermore each $m \in D\left(E^{\prime}\right)$ generates an associative *-algebra $\mathfrak{U}(m)$ in $\mathfrak{M}^{(2)}$ : $m \in D\left(E^{\prime}\right), x, y \in E$ imply

$$
(m(x) m(y))_{v \mu}=\sum_{j=1}^{N_{v} \wedge N_{\mu}} m(x)_{v j} m(y)_{j \mu}
$$

e.g. $(m(x) m(y))_{v \mu}=0$ if $\mu>\max \left\{N_{1}, \ldots, N_{v}\right\}=N_{v}^{(1)}$; thus $m(x) m(y)$ is a matrix of the same class as $m(x)$, therefore all products $m\left(x_{1}\right) \ldots m\left(x_{n}\right)$ are well defined and are associative and satisfy the right continuity properties $(10)$ :

$$
x_{1} \otimes \ldots \otimes x_{n} \mapsto\left(\sum_{\nu=1}^{\infty}\left|\left(m\left(x_{1}\right) \ldots m\left(x_{n}\right)\right)_{v \mu}\right|^{2}\right)^{1 / 2}
$$

is a continuous seminorm because there are really only finite sums involved. The same holds if different $m_{j} \in D\left(E^{\prime}\right)$ are considered. Well known examples of this class 
are the matrix realizations of the canonical commutation relations, multiplied by $f \in E^{\prime}$ :

$$
m(x)=a(x)+a^{*}(x), \quad a(x)_{v \mu}=f(x) \delta_{v+1, \mu} v^{1 / 2}, \quad v, \mu \in \mathbb{N} .
$$

This shows that the class $D\left(E^{\prime}\right)$ contains matrix representations of unbounded operators.

As a last immediate application of Theorem 1.2 we treat a recursion relation connecting various $n$-point-functionals $T_{n}$ for a given $T=\left\{1, T_{1}, T_{2}, \ldots\right\} \in \underline{E}_{+, 1}^{\prime}$. By the theorem we have for some $m \in \mathfrak{M}_{\infty}^{(2)}\left(E^{\prime}\right)$

$$
T_{1}=m_{00}, \quad T_{n+1}=\sum_{j_{1} \ldots j_{n}=0}^{\infty} m_{0 j_{1}} \otimes m_{j_{1} j_{2}} \otimes \ldots \otimes m_{j_{n} 0} \quad n=1,2,3, \ldots
$$

with convergence properties specified above. These convergence properties allow to resum these expressions to get

$$
\begin{aligned}
& T_{1}=\hat{T}_{1} \\
& T_{n}=\hat{T}_{n}+\sum_{k=2}^{n} \sum_{\underline{v}_{k} \in P_{k}(n)} \hat{T}_{v_{1}} \otimes \ldots \otimes \hat{T}_{v_{k}} \equiv \hat{T}_{n}+T_{n}^{(0)} \quad n=2,3,4, \ldots
\end{aligned}
$$

where

$$
\begin{aligned}
& \hat{T}_{n+1}=\sum_{j_{1} \ldots j_{n}=1}^{\infty} m_{0 j_{1}} \otimes m_{j_{1} j_{2}} \otimes \ldots \otimes m_{j_{n} 0} \quad n=1,2,3, \ldots \\
& P_{k}(n)=\left\{\underline{v}_{k}=\left(v_{1}, \ldots, v_{k}\right) ; v_{j} \in \mathbb{N}, \sum_{j=1}^{k} v_{j}=n\right\} .
\end{aligned}
$$

This yields

$$
\begin{aligned}
& T=T^{(0)}+\hat{T}=\left\{1, T_{1}, T_{2}^{(0)}, T_{3}^{(0)}, \ldots\right\}+\left\{0,0, \hat{T}_{2}, \hat{T}_{3}, \ldots\right\} \\
& \hat{T}\left\lceil\underline{E}_{+} \cap\left(\{0\} \oplus \bigoplus_{n=1}^{\infty} E_{n}\right) \geqq 0\right.
\end{aligned}
$$

and implies a lot of inequalities for $\hat{T}$, in particular those corresponding to (4) and the most simple one's are

$$
\left|\hat{T}_{n+m}\left(x_{n}^{*} \otimes x_{m}\right)\right|^{2} \leqq \hat{T}_{2 n}\left(x_{n}^{*} \otimes x_{n}\right) \cdot \hat{T}_{2 m}\left(x_{m}^{*} \otimes x_{m}\right)
$$

for all $x_{n} \in E_{n}, x_{m} \in E_{m}, n, m=1,2, \ldots$.

A useful application of these relations is a condition on $T \in \underline{E}_{+, 1}^{\prime}$ to be trivial in the sense, that all $T_{n}(n>N)$ are determined by $T_{1}, \ldots, T_{N}$. We treat the case $N=2$.

Corollary 1.4. $T \in \underline{E}_{+, 1}^{\prime}$ is uniquely determined by $T_{1}$ and $T_{2}$ if for some $n \in \mathbb{N}$ the equation $\hat{T}_{2 n}=0$ holds.

Proof. Suppose $\hat{T}_{2 n}=0$, then by $\left(21^{\prime}\right) \hat{T}_{n+m}=0$ for all $m=1,2, \ldots$; that is if $n=1$, $\hat{T}_{m}=0$ for all $m=2,3, \ldots$ and thus by $(20) T_{m}=T_{1}^{\otimes m}, m=1,2, \ldots$; if $n \geqq 2$ then $\hat{T}_{m}=0$ for all $m=3,4, \ldots$ and thus for all $m=3,4, \ldots$

$$
T_{m}=\sum_{k=2}^{m} \sum_{\substack{\underline{v}_{k} \in P_{k}(m) \\ v_{j} \leqq 2}} \hat{T}_{v_{1}} \otimes \ldots \otimes \hat{T}_{v_{k}} .
$$


Remark. (a) A natural generalization of Corollary 1.4 would be to answer the question when a $T \in E_{+, 1}^{\prime}$ is uniquely determined by $T_{1}, T_{2}, \ldots, T_{N}, N>2$. We will discuss this problem in Part II.

(b) Within a QFT in the sense of Wightman such a statement as in Corollary 1.4 is known to hold $[11,14]$. There one has to use the truncated $2 n$-pointfunctionals $W_{2 n}^{t}$ instead of $\hat{T}_{2 n}$.

\section{On a Special Kind of Extension Problems for Monotone Continuous Linear Functionals}

The kind of extension problems we have in mind has been described in the introduction; we ask which conditions make a given set $T_{(2 N)}=\left\{1, T_{1}, T_{2}, \ldots, T_{2 N}\right\}$, $T_{n} \in E_{n}^{\prime}, n=1, \ldots, 2 N$, of $n$-point-functionals to be the first $2 N+1 n$-pointfunctionals of a m.c.l. functional $T$ on $E$. Obviously a necessary condition for the existence of at least one $T \in \underline{E}_{+, 1}^{\prime}$ such that $T \nmid \underline{E}_{(2 N)}=T_{(2 N)}$ is

$$
T_{(2 N)} \nmid \underline{E}_{+} \cap \underline{E}_{(2 N)} \geqq 0
$$

e.g. monotonicity of $T_{(2 N)}$ on that part of $E_{+}$where it can be such. We use the term $T_{(2 N)} \geqq 0$ to characterize monotonicity of $T_{(2 N)} \in E_{(2 N)}^{\prime}$ according to (22).

The most general criterium for monotone continuous linear extensions is that of Bauer and Namioka [3, 7]. It seems to be not very useful in this context. The first step in attacking these extension problems is to exploit the above monotonicity condition as much as possible. This can as we think be done best by deriving a representation for $T_{(2 N)}$ which is as close to those for m.c.l. functionals as it can be. The method we use therefore is very similar to that in Theorem 1.2.

Theorem 2.1. a) Assume the hypotheses of Theorem 1.2 on E. Then a continuous linear functional $T_{(2 N)}=\left\{1, T_{1}, \ldots, T_{2 N}\right\}$ on $\underline{E}_{(2 N)}=\bigoplus_{n=0}^{2 N} E_{n}$ is monotone in the sense of inequality (22) if and only if there is a nuclear linear function $\Phi_{(N)}$ from $\underline{E}_{(N)}$ onto some separable pre-Hilbertspace $V_{N}=\left(\Phi_{(N)}\left(\underline{E}_{(N)}\right),\langle\cdot, \cdot\rangle_{(N)}\right)$ such that

$$
T_{(2 N)}\left(\underline{x}^{*} \cdot \underline{y}\right)=\left\langle\Phi_{(N)}(\underline{x}), \Phi_{(N)}(\underline{y})\right\rangle_{(N)} \quad \text { all } \quad \underline{x}, \underline{y} \in \underline{E}_{(N)}
$$

and $\Phi_{(N)}$ satisfies the following consistency relation

$$
\left\langle\Phi_{(N)}\left(\underline{x}_{1}\right), \Phi_{(N)}\left(\underline{y}_{1}\right)\right\rangle_{(N)}=\left\langle\Phi_{(N)}\left(\underline{x}_{2}\right), \Phi_{(N)}\left(\underline{y}_{2}\right)\right\rangle_{(N)}
$$

for all $\underline{x}_{j}, \underline{y}_{j} \in \underline{E}_{(N)}$ such that $\underline{x}_{1}^{*} \cdot \underline{y}_{1}=\underline{x}_{2}^{*} \cdot \underline{y}_{2}$.

b) Any other such realization of $T_{(2 N)}$ is obtained from a special one by means of a unitary transformation. In particular there is such a realization $\left(\underline{\tau}_{(N)}\left(\underline{E}_{(N)}\right),\langle\cdot, \cdot\rangle_{2}\right)$ in which $\underline{\tau}_{(N)}\left(\underline{E}_{(N)}\right)$ is a dense subspace of some Hilbertspace $l^{2}$ of square summable sequences (nuclear representation of $T_{(2 N)}$ ).

Proof. (a) The sufficiency of the above representation for a continuous linear functional to be monotone is obvious: Each $\underline{\underline{\xi}} \in \underline{E}_{+} \cap \underline{E}_{(2 N)}$ has a representation 
$\underline{\xi}=\sum_{j=1}^{m} \underline{x}_{j}^{*} \cdot \underline{x}_{j}, \underline{x}_{j} \in \underline{E}_{(N)} ;$ thus we get by Equation (23)

$$
T_{(2 N)}(\underline{\xi})=\sum_{j=1}^{m} T_{(2 N)}\left(\underline{x}_{j}^{*} \cdot \underline{x}_{j}\right)=\sum_{j=1}^{m}\left\langle\Phi_{(N)}\left(\underline{x}_{j}\right), \Phi_{(N)}\left(\underline{x}_{j}\right)\right\rangle_{(N)}
$$

e.g. inequality (22).

(b) For the converse note that by (22) and L. 1.1

$$
\underline{x} \mapsto q_{(N)}(\underline{x})=T_{(2 N)}\left(\underline{x}^{*} \cdot \underline{x}\right)^{1 / 2}
$$

defines a continuous seminorm on the nuclear space $\underline{E}_{(N)}$; therefore the canonical quotient map

$$
\Phi_{(N)}: \underline{E}_{(N)} \rightarrow \underline{E}_{(N)} / q_{(N)}^{-1}(0) \equiv \Phi_{(N)}\left(\underline{E}_{(N)}\right)
$$

is nuclear and $\left\langle\Phi_{(N)}(\underline{x}), \Phi_{(N)}(\underline{y})\right\rangle_{(N)}=T_{(2 N)}(\underline{x} * \underline{y})$ defines a scalarproduct on $\Phi_{(N)}\left(\underline{E}_{(N)}\right)$ such that $(23)$ holds. By definition $\Phi_{(N)}$ is linear and satisfies the consistency relation (24).

(c) If $\left(\Psi_{(N)}\left(\underline{E}_{(N)}\right),(., .)_{(N)}\right)$ is another such realization of $T_{(2 N)}$, define a linear map according to

$$
\begin{aligned}
& U: \Phi_{(N)}\left(\underline{E}_{(N)}\right) \rightarrow \Psi_{(N)}\left(\underline{E}_{(N)}\right), \\
& U \Phi_{(N)}(\underline{x})=\Psi_{(N)}(\underline{x}) \text { for all } \underline{x} \in \underline{E}_{(N)} .
\end{aligned}
$$

Because of Equation (23) $U$ is well defined, surjective, and isometric and thus extends to a unitary map (of the completions of the above pre-Hilbertspaces).

(d) Again there is an orthonormal basis $\varphi_{j}=\Phi_{(N)}\left(\underline{x}_{j}\right), j=0,1, \ldots$ in $\Phi_{(N)}\left(\underline{E}_{(N)}\right)$. Therefore we get

$$
\begin{aligned}
\Phi_{(N)}(\underline{x}) & =\sum_{j=0}^{\infty} \tau_{j}(\underline{x}) \varphi_{j} \quad \text { in } \quad V_{N} \\
\underline{x} & =\sum_{j=0}^{\infty} \tau_{j}(\underline{x}) \underline{x}_{j} \quad \text { in } \quad\left(\underline{E}_{(N)}, q_{(N)}\right) \\
\tau_{j}(\underline{x}) & =\left\langle\varphi_{j}, \Phi_{(N)}(\underline{x})\right\rangle_{(N)} \quad \text { all } \quad \underline{x} \in \underline{E}_{(N)} .
\end{aligned}
$$

Define

$$
\underline{\tau}_{(N)}(\underline{x})=\left\{\tau_{1}(\underline{x}), \tau_{2}(\underline{x}), \ldots\right\}
$$

then clearly $\underline{\tau}_{(N)}(\underline{x}) \in l^{2}$ and

$$
\left\langle\underline{\tau}_{(N)}(\underline{x}), \underline{\tau}_{(N)}(\underline{y})\right\rangle_{2}=\left\langle\Phi_{(N)}(\underline{x}), \Phi_{(N)}(\underline{y})\right\rangle_{(N)},
$$

thus $U \Phi_{(N)}(\underline{x})=\underline{\tau}_{(N)}(\underline{x})$ defines a unitary map $U: \Phi_{(N)}\left(\underline{E}_{(N)}\right) \rightarrow \underline{\tau}_{(N)}\left(\underline{E}_{(N)}\right)$ and thus $\underline{\tau}_{(N)}=U \Phi_{(N)}$ is again nuclear.

It is convenient to introduce the following abbreviation.

Definition 2.2. Suppose $T_{(2 N)} \in \underline{E}_{(2 N)}^{\prime}$ satisfies $T_{(2 N)} \geqq 0$. If $\Phi_{(N)}$ denotes the canonical quotient map according to (25) and (26) we call the representation

$$
V_{N}=\left(\Phi_{(N)}\left(\underline{E}_{(N)}\right),\langle\cdot, \cdot\rangle_{(N)}\right)
$$

such that (23) and (24) hold the canonical pre-Hilbertspace realization of $T_{(2 N)}$. 
Proposition 2.3. a) The canonical pre-Hilbertspace realization of a given $T_{(2 N)} \in E_{(2 N)}^{\prime}$ $T_{(2 N)} \geqq 0$, allows to define a linear map $A_{(N-1)}: E_{1} \rightarrow L\left(V_{N-2}, V_{N-1}\right), V_{n}=\Phi_{(N)}\left(\underline{E}_{(n)}\right)$, $n=1, \ldots, N$ such that

i) $A_{(N-1)}(x) \Phi_{(N)}(\underline{y})=\Phi_{(N)}(x \cdot \underline{y})$ all $x \in E_{1}$, all $\underline{y} \in \underline{E}_{(N-2)}$

ii) $A_{(N-1)}(x)^{*} \uparrow V_{N-2}=A_{(N-1)}\left(x^{*}\right) \uparrow V_{N-2}$ all $x \in E_{1}$.

b) If $T_{(2 N)}$ satisfies in addition

$$
\left|T_{(2 N)}\left(\underline{y}^{*} \cdot \underline{x}\right)\right| \leqq q_{(N+1)}(\underline{y}) T_{(2 N)}\left(\underline{x}^{*} \cdot \underline{x}\right)^{1 / 2}
$$

for all $\underline{x} \in \underline{E}_{(N-1)}$, all $\underline{y} \in \underline{E}_{(N+1)}, q_{(N+1)}$ some continuous seminorm on $\underline{E}_{(N+1)}$, the map $A_{(N-1)}$ has a linear extension $A_{(N)}: E_{1} \rightarrow L\left(V_{N-1}, V_{N}\right)$ such that (i) and (ii) of part a) hold on $V_{N-1}$ and in addition

iii) $\Phi_{0}=\Phi_{(N)}(\underline{1})$ is cyclic for $A_{(N)}\left(E_{1}\right)$ in $\mathscr{H}_{(N)}=\tilde{V}_{N}$.

Proof. a) We show that (i) can be used to define $A_{(N-1)}$. For this we have to show that $\Phi_{(N)}(\underline{y})=0, \underline{y} \in \underline{E}_{(N-2)}$, implies $\Phi_{(N)}(x \cdot \underline{y})=0$ for all $x$ in $E_{1}$. By $(24)$ we easily get for all $x \in E_{1}$ :

$$
\left\|\Phi_{(N)}(x \cdot \underline{y})\right\|_{(N)}^{2}=\left\langle\Phi_{(N)}\left(x^{*} \cdot x \cdot \underline{y}\right), \Phi_{(N)}(\underline{y})\right\rangle_{(N)}=0 .
$$

Thus $A_{(N-1)}(x)$ is well defined on $V_{N-2}$ by Equation (i). The properties of $\Phi_{(N)}$ easily yield those claimed for $A_{(N-1)}$. The consistency relation again yields (ii).

b) If inequality (28) holds the definition

$$
A_{(N)}(x) \Phi_{(N)}(\underline{y})=\Phi_{(N)}(x \cdot \underline{y})
$$

works on $V_{N-1}$, because $\Phi_{(N)}(\underline{y})=0, \underline{y} \in \underline{E}_{(N-1)}$, implies

$$
\left\|\Phi_{(N)}(x \underline{y})\right\|_{(N)}^{2} \leqq q_{(N+1)}\left(x^{*} \cdot x \cdot \underline{y}\right) \cdot\left\|\Phi_{(N)}(\underline{y})\right\|_{(N)}=0 .
$$

Clearly $A_{(N)}$ is a linear extension of $A_{(N-1)}$ and satisfies (i) and (ii) above. By definition of $A_{(N)}$ we get

$$
\begin{aligned}
\mathscr{D}_{1} & =\mathbb{C} \Phi_{0}, \\
\mathscr{D}_{n+1} & =\operatorname{lin}\left(\mathscr{D}_{n} \cup A_{(N)}\left(E_{1}\right) \mathscr{D}_{n}\right), \quad n=1, \ldots, N ; \\
\mathscr{D}_{N+1} & =\Phi_{(N)}\left(\bigoplus_{n=0}^{N} E_{1}^{\otimes n}\right)
\end{aligned}
$$

which is dense in $V_{N}$ and thus in $\mathscr{H}_{(N)}$. Therefore (iii) holds.

Remark. Clearly there are some other obviously necessary conditions on $T_{(2 N)}$ for the existence of at least one m.c.l. extension:

$$
\left|T_{(2 N)}\left(\underline{y}_{(n)}^{*} \underline{x}_{(m)}\right)\right| \leqq q_{(n)}\left(\underline{y}_{(n)}\right) \cdot T_{(2 N)}\left(\underline{x}_{(m)}^{*} \cdot \underline{x}_{(m)}\right)^{1 / 2}
$$

all $\underline{x}_{(m)} \in \underline{E}_{(m)}, \underline{y}_{(n)} \in \underline{E}_{(n)}, m=1, \ldots, N, n=2 N-m, q_{(n)}$ some continuous seminorm on $\underline{E}_{(n)}$. 
The validity of these inequalities is equivalent to the existence of some continuous linear functions $\Psi_{(N)}^{(n)}: \underline{E}_{(n)} \rightarrow \mathscr{H}_{(N)}, n=N, \ldots, 2 N$, such that

(i) $T_{(2 N)}\left(\underline{y}^{*} \cdot \underline{x}\right)=\left\langle\Psi_{(N)}^{(n)}(\underline{y}), \Phi_{(N)}(\underline{x})\right\rangle_{(N)}$

(ii) $\Psi_{(N)}^{(N)}=\Phi_{(N)}$

(iii) $\left\|\Psi_{(N)}^{(n)}(\underline{y})\right\|_{(N)} \leqq q_{(n)}(\underline{y})$

for all

$\underline{y} \in \underline{E}_{(n)}, \quad$ all $\quad \underline{x} \in \underline{E}_{(m)}, \quad n=N, \ldots, 2 N, \quad m=2 N-n$.

Suppose that a given $T_{(2 N)} \in \underline{E}_{(2 N)}^{\prime}, T_{(2 N)} \geqq 0$, with canonical pre-Hilbertspace realization $V_{N}=\left(\Phi_{(N)}\left(\underline{E}_{(N)}\right),\langle\cdot, \cdot\rangle_{(N)}\right)$ has a m.c.l. extension $T \in \underline{E}_{+, 1}^{\prime}$. This $T$ has a canonical pre-Hilbertspace realization $V_{T}=\left(\Phi_{T}(E),\langle\cdot, \cdot\rangle_{T}\right)$ and thus a canonical isometry $J: \mathscr{H}_{(N)} \rightarrow \mathscr{H}_{T}$ from $\mathscr{H}_{(N)}$ into $\mathscr{H}_{T}$ is well defined by continuous linear extension of

$$
J \Phi_{(N)}(\underline{x})=\Phi_{T}(\underline{x}) \quad \text { all } \quad \underline{x} \in \underline{E}_{(N)}
$$

because of

$$
\left\|J \Phi_{(N)}(\underline{x})\right\|_{T}^{2}=T\left(\underline{x}^{*} \cdot \underline{x}\right)=T_{(2 N)}\left(\underline{x}^{*} \cdot \underline{x}\right)=\left\|\Phi_{(N)}(\underline{x})\right\|_{(N)}^{2} .
$$

In terms of the 'fields' $A_{T}$ and the 'candidates for the fields' $A_{(N)}$ we may formulate the extension problem as follows:

$T_{(2 N)} \in \underline{E}_{(2 N)}^{\prime}, T_{(2 N)} \geqq 0$, has a m.c.l. extension $T$ iff there exists

(i) a separable Hilbertspace $\mathscr{H}=\mathscr{H}_{T}$ in which a 'field' $A=A_{T}$ :

$E_{1} \rightarrow L\left(\mathscr{D}_{A}, \mathscr{D}_{A}\right)$ acts according to Equations (15) and

(ii) a canonical isometry $J: \mathscr{H}_{(N)} \rightarrow \mathscr{H}$ such that

$$
J A_{(N)}\left(x_{1}\right) \ldots A_{(N)}\left(x_{n}\right) \Phi_{(N)}(\underline{1})=A\left(x_{1}\right) \ldots A\left(x_{n}\right) J \Phi_{(N)}(\underline{1})
$$

holds for all $x_{j} \in E_{1}$ and $n=0,1, \ldots, N$.

Thus we see that the construction of a m.c.l. extension of $T_{(2 N)} \geqq 0$ requires two main steps:

1. The construction of some separable Hilbertspace $\mathscr{H}$ in which we can embed $\mathscr{H}_{(N)}$ isometrically.

2. The construction of some linear function $A: E_{1} \rightarrow L\left(\mathscr{D}_{A}, \mathscr{D}_{A}\right), \mathscr{D}_{A}$ dense in $\mathscr{H}$, which satisfies Equations (15) and (32).

In this generality an enormous amount of freedom is involved in this problem. So we propose first to look for a special kind of extensions thus making the extension problem much more definite. In order to do this we introduce

Definition 2.4. $T \in \underline{E}_{+, 1}^{\prime}$ is called a minimal extension of $T_{(2 N)} \in \underline{E}_{(2 N)}^{\prime}, T_{(2 N)} \geqq 0$, if and only if

(i) $T\urcorner \underline{E}_{(2 N)}=T_{(2 N)}$

(ii) The Hilbertspace $\mathscr{H}_{T}=\tilde{V}_{T}$ of the canonical pre-Hilbertspace realization $V_{T}=\left(\Phi_{T}(E),\langle\cdot, \cdot\rangle_{T}\right)$ of $T$ and the Hilbertspace $\mathscr{H}_{(N)}=\tilde{V}_{(N)}$ of the canonical pre- 
Hilbertspace realization $V_{(N)}=\left(\Phi_{(N)}\left(\underline{E}_{(N)}\right),\langle\cdot, \cdot\rangle_{(N)}\right)$ are canonically isomorphic (e.g. $J$ as defined by (31) is a unitary map from $\mathscr{H}_{(N)}$ onto $\left.\mathscr{H}_{T}\right)$.

A second step then has to treat the case of more general extensions using the knowledge of minimal extensions.

Remark. Some comments on the relevance of this special kind of extensions seem to be in order.

a) To look for minimal extensions seems to be the only way to get necessary and sufficient conditions on $T_{(2 N)}$ for the existence of (at least minimal) m.c.l. extensions. We will show this in this section.

b) The known examples in relativistic QFT (generalized free fields) are constructed according to the strategy proposed above. This will be shown in Section 3.

c) For the simplest case $E \cong \mathbb{C}$ all m.c.l. extensions can be constructed according to this procedure (Section 4).

d) There are cases of $T_{(2 N)}$ where only minimal extensions exist. This is shown in Part II.

e) In application to QFT the construction of minimal extensions is the easiest way of constructing m.c.l. extensions which are Poincaré-covariant and satisfy the spectral-condition (Part II).

f) The notion of minimal extensions allows to specify a case where a relativistic QFT is fixed in terms of finitely many vacuum expectation values (Part II).

g) If the usual coupling of fields and additive quantum numbers is assumed (for instance the case of charged fields) one easily sees that minimal extensions do not describe the situation one expects (this has been pointed out by O. Steinmann).

Now we want to give arguments which support statement a) above. If we look for minimal extensions $T$ of $T_{(2 N)}$ we can always identify $\mathscr{H}_{T}$ and $\mathscr{H}_{(N)}$; e.g. Step 1. of the general case disappears and thus we get

Proposition 2.5. A functional $T_{(2 N)} \in \underline{E}_{(2 N)}^{\prime}$ which satisfies inequalities (22) and (28) has a minimal extension $T \in \underline{E}_{+, 1}^{\prime}$ if and only if the linear function $A_{(N)}: E_{1} \rightarrow L\left(V_{N-1}, V_{N}\right)$ which realizes $T_{(2 N)}$ according to Proposition $\left.2.3 b\right)$ has a symmetric linear extension $A: E_{1} \rightarrow L\left(\mathscr{D}_{A}, \mathscr{D}_{A}\right)$ such that

(i) $\Phi_{0}=\Phi_{(N)}(1) \in \mathscr{D}_{A}, \overline{\mathscr{D}}_{A}=\mathscr{H}_{(N)}$,

$$
\mathscr{D}_{A}=\bigcup_{n=1}^{\infty} D_{n}, D_{1}=\mathbb{C} \Phi_{0}, D_{n+1}=\operatorname{lin}\left(D_{n} \cup A\left(E_{1}\right) D_{n}\right),
$$

(ii) $A(x) * \uparrow \mathscr{D}_{A}=A\left(x^{*}\right) \uparrow \mathscr{D}_{A}$,

(iii) $p_{n}\left(x_{1} \otimes \ldots \otimes x_{n}\right)=\left\|A\left(x_{1}\right) \ldots A\left(x_{n}\right) \Phi_{0}\right\|_{(N)}$ are continuous seminorms on $E_{1}^{\otimes n}$, $n=1,2, \ldots$

(iv) $A\left(x_{1}\right) \ldots A\left(x_{n}\right) \Phi_{0}=A_{(N)}\left(x_{1}\right) \ldots A_{(N)}\left(x_{n}\right) \Phi_{0}$ for all $x_{j} \in E_{1}, n=1, \ldots, N$.

Proof. If $T \in \underline{E}_{+, 1}^{\prime}$ is a minimal extension of $T_{(2 N)}$ we know $\mathscr{H}_{T}$ and $\mathscr{H}_{(N)}$ to be canonically isomorphic. Thus the identification of $\mathscr{H}_{T}$ and $\mathscr{H}_{(N)}$ yields a linear function $A: E_{1} \rightarrow L\left(\mathscr{D}_{A}, \mathscr{D}_{A}\right)$ which has the properties as specified above. Conversely suppose we are given a linear function $A: E_{1} \rightarrow L\left(\mathscr{D}_{A}, \mathscr{D}_{A}\right)$ as above. 
This then defines a $T \in \underline{E}_{+, 1}^{\prime}$ according to

$$
T_{n}\left(x_{1} \otimes \ldots \otimes x_{n}\right)=\left\langle\Phi_{0}, A\left(x_{1}\right) \ldots A\left(x_{n}\right) \Phi_{0}\right\rangle_{(N)}
$$

for all $x_{j} \in E_{1}$ and all $n=1,2, \ldots$.

By (ii) and (iv) we see $T \uparrow \underline{E}_{(2 N)}=T_{(2 N)}$. $\mathscr{H}_{T}$ is canonically isomorphic to $\mathscr{H}_{(N)}$ by construction. Therefore this $T \in \underline{E}_{+, 1}^{\prime}$ is a minimal extension of $T_{(2 N)}$.

Remark. Even the case of minimal extensions includes in general the problem of extending a not densely defined operator $A_{(N)}(x)$ to a densely defined operator $A(x), x \in E_{1}$. This is definitely the case when $V_{N-1}$ is not dense in $\mathscr{H}_{(N)}$. Later (Part II) we will discuss necessary and sufficient conditions on $T_{(2 N)}$ such that $V_{N-1}$ is dense in $\mathscr{H}_{(N)}$.

A natural question is to ask how many minimal extensions a given $T_{(2 N)} \geqq 0$ may have and how these are specified. In order to give a preliminary answer we proceed as follows. Suppose $0 \leqq T_{(2 N)} \in \underline{E}_{(2 N)}^{\prime}$ has a minimal extension $T \in \underline{E}_{+, 1}^{\prime}$. Then there is a "field" $A: E_{1} \rightarrow L\left(\mathscr{D}_{A}, \mathscr{D}_{A}\right)$ according to Proposition 2.5 and in particular we may define a chain of linear functions $\left(D_{n}\right.$ as in Proposition 2.5 (i))

$$
\begin{aligned}
& A_{(n)}: E_{1} \rightarrow L\left(D_{n}, D_{n+1}\right) \\
& A_{(n)}(x)=A(x)\left\lceil D_{n} \quad n=1,2, \ldots .\right.
\end{aligned}
$$

Then for suitable $M \in\{1, \ldots, N\}$ and $n \geqq M+1 A_{(n)}(x)$ is densely defined in $\mathscr{H}_{(N)}$ and thus has a unique adjoint in $\mathscr{H}_{(N)}$ such that

$$
A(x)^{*} \subseteq A_{(n+1)}(x)^{*} \subseteq A_{(n)}(x)^{*} \quad n=M+1, M+2, \ldots
$$

because of $A_{(n)}(x) \subseteq A_{(n+1)}(x) \subseteq A(x)$ for all $x \in E_{1}$ and $n=1,2, \ldots$.

Property (ii) of Proposition 2.5 then implies

$$
\begin{aligned}
& A_{(n)}\left(x^{*}\right)=A_{(n)}(x)^{*} \uparrow D_{n} \quad n \geqq M+1 \\
& A_{(n+1)}(x)=A_{(n)}\left(x^{*}\right) * \uparrow D_{n+1} \quad n \geqq M+1
\end{aligned}
$$

By property (iii) we have in addition that

$$
p_{n}\left(x_{1} \otimes \ldots \otimes x_{n}\right)=\left\|A_{(n)}\left(x_{1}\right) \ldots A_{(n)}\left(x_{n}\right) \Phi_{0}\right\|_{(N)}
$$

defines a continuous seminorm on $E_{1}^{\otimes n}$ for all $n=M+1, M+2, \ldots$.

This essentially shows

Corollary 2.6. Suppose $T_{(2 N)} \in \underline{E}_{(2 N)}^{\prime}, \quad T_{(2 N)} \geqq 0$, has minimal extensions. Define $M \in\{1, \ldots, N\}$ to be minimal such that $V_{M}=\Phi_{(N)}\left(E_{(M)}\right)$ is dense in $\mathscr{H}_{(N)}$. Then each minimal extension of $T_{(2 N)}$ is fixed by the definition of $A_{(M+1)}(x)$ on $D_{M+1}$ $=\Phi_{(N)}\left(\bigoplus_{n=0}^{M} E_{1}^{\otimes n}\right)$. In particular if $M<N T_{(2 N)}$ has at most one minimal extension and if $M=N$ each minimal extension of $T_{(2 N)}$ is fixed by fixing a $2 N+1$-pointfunctional $T_{2 N+1}$ appropriately.

Notice that the relations (34) express a construction procedure for the functions $A_{(n)}$. These relations yield a chain of inequalities which are in principle inequalities for $T_{(2 N)}$ respectively $T_{2 N+1}$. This is used to prove 
Theorem 2.7. For a given $T_{(2 N)} \in \underline{E}_{(2 N)}^{\prime}, T_{(2 N)} \geqq 0$, let us distinguish

Case $A . V_{N-1}$ is not dense in $\mathscr{H}_{(N)}$.

Case B. $V_{M}$ is dense in $\mathscr{H}_{(N)}, M \leqq N-1$.

Then in Case $A T_{(2 N)}$ has a minimal extension which is fixed by a given $2 N+1$-pointfunctional $T_{2 N+1}=T_{2 N+1}^{*} \in E_{2 N+1}^{\prime}$ if and only if

a) there is a continuous seminorm $q_{(N+1)}$ on $\underline{E}_{(N+1)}$ such that

$$
\left.\left|T_{(2 N+1)}\left(\underline{y}^{*} \cdot z \cdot \underline{x}\right)\right| \leqq T_{(2 N}\right)\left(\underline{y}^{*} \cdot \underline{y}\right)^{1 / 2} \cdot q_{(N+1)}(z \cdot \underline{x})
$$

for all $\underline{x}, \underline{y} \in \underline{E}_{(N)}$ and all $z \in E_{1}, T_{(2 N+1)}=\left\{1, T_{1}, \ldots, T_{2 N}, T_{2 N+1}\right\}$,

b) the following successively defined chain of inequalities holds

$$
\begin{aligned}
& \quad\left\langle A_{(n)}(x) \Psi_{(n)}, A_{(n)}\left(x_{1}\right) \ldots A_{(n)}\left(x_{k}\right) \Phi_{0}\right\rangle_{(N)} \mid \\
& \quad \leqq\left\|\Psi_{(n)}\right\|_{(N)} \cdot p_{k+1}\left(x \otimes x_{1} \otimes \ldots \otimes x_{k}\right) \quad\left(K_{n, k}\right)
\end{aligned}
$$

for all $x, x_{j} \in E_{1}$, all $\Psi_{(n)} \in D_{n}, p_{n}$ are continuous seminorms on $E_{1}^{\otimes n}, k=n$ and $k=n-1, n=N+1, N+2, \ldots ;$ the $A_{(n)}$ are defined according to Equation (34).

In Case $B T_{(2 N)}$ has a minimal extension if and only if the inequalities $\left(K_{n, k}\right)$ hold for $n=N, N+1, \ldots$.

By straightforward insertion of an orthonormal basis $\left\{\varphi_{j}\right\}_{j \in \mathbb{N}} \subset V_{N}$ of $\mathscr{H}_{(N)}$ into $\left(K_{n, k}\right)$ these inequalities are inequalities for $T_{(2 N)}$ respectively $T_{(2 N+1)}$.

Proof. By Proposition 2.5 and Corollary 2.6 it is immediate that the inequalities (35) and $\left(K_{n, k}\right), n \geqq N+1$ respectively $\left(K_{n, k}\right), n \geqq N$ are necessary.

Conversely assume that in Case A there is a $T_{2 N+1}=T_{2 N+1}^{*} \in E_{2 N+1}^{\prime}$ such that (35) holds; then by Riesz-Fréchet there is exactly one linear function

$$
\Psi_{(N)}^{N+1}: \underline{E}_{(N+1)} \rightarrow \mathscr{H}_{(N)}
$$

such that

$$
\begin{aligned}
& T_{(2 N+1)}\left(\underline{y}^{*} \cdot z \cdot \underline{x}\right)=\left\langle\Phi_{(N)}(\underline{y}), \Psi_{(N)}^{N+1}(z \cdot \underline{x})\right\rangle_{(N)}=\left\langle\Psi_{(N)}^{N+1}(z \cdot \underline{y}), \Phi_{(N)}(\underline{x})\right\rangle_{(N)} \\
& \left\|\Psi_{(N)}^{N+1}(z \cdot \underline{x})\right\|_{(N)} \leqq q_{(N+1)}(z \cdot \underline{x})
\end{aligned}
$$

holds for all $\underline{x}, \underline{y} \in \underline{E}_{(N)}$ and all $z \in E_{1}$. Using (36) we see that a linear map

$$
A_{(N+1)}: E_{1} \rightarrow L\left(V_{N}, \mathscr{H}_{(N)}\right)
$$

is well-defined by

$$
A_{(N+1)}(x) \Phi_{(N)}(\underline{y})=\Psi_{(N)}^{N+1}(x \cdot \underline{y}) \quad x \in E_{1}, \quad \underline{y} \in \underline{E}_{(N)}
$$

and satisfies

$$
A_{(N+1)}(x)^{*} \uparrow V_{N}=A_{(N+1)}\left(x^{*}\right) \uparrow V_{N} \quad x \in E_{1}
$$

Obviously $A_{(N+1)}$ is strongly continuous. In particular $A_{(N+1)}(x)$ is a densely defined linear operator in $\mathscr{H}_{(N)}$ for all $x \in E_{1}$. By inequality $K_{N+1, N+1}$ we see

$$
D_{N+2}=\operatorname{lin}\left(D_{N+1} \cup A_{(N+1)}\left(E_{1}\right) D_{N+1}\right) \subseteq D\left(A_{(N+1)}\left(x^{*}\right)^{*}\right)
$$

thus

$$
A_{(N+2)}(x)=A_{(N+1)}\left(x^{*}\right)^{*} \uparrow D_{N+2}
$$


is welldefined and satisfies

$$
A_{(N+2)}(x) \uparrow D_{N+1}=A_{(N+1)}(x) \uparrow D_{N+1}
$$

because of (37b). According to $K_{N+1, N+1}$

$$
p_{N+2}\left(x \otimes x_{1} \ldots \otimes x_{N+1}\right)=\left\|A_{(N+2)}(x) A_{(N+2)}\left(x_{1}\right) \ldots A_{(N+2)}\left(x_{N+1}\right) \Phi_{0}\right\|_{(N)}
$$

is a continuous seminorm on $E_{1}^{\otimes(N+2)}$. Inequality $K_{N+2, N+1}$ implies $D_{N+2} \subseteq D\left(A_{(N+2)}\left(x^{*}\right)^{*}\right)$ for all $x \in E_{1}$ and therefore

$$
A_{(N+2)}\left(x^{*}\right)^{*} \uparrow D_{N+2}=A_{(N+1)}\left(x^{*}\right) * \uparrow D_{N+2}=A_{(N+2)}(x) \text { for all } x \in E_{1} .
$$

Similarly we get by induction using the whole chain of inequalities $K_{n, k}$, $k=n, n-1 ; n \geqq N+1$ a chain of linear functions $A_{(n)}: E_{1} \rightarrow L\left(D_{n}, D_{n+1}\right)$, $n=N+1, N+2, \ldots, D_{N+1}=\mathscr{D}_{N+1}$, such that Equation (34) and

$$
A_{(N+1)}(x) \subseteq A_{(N+2)}(x) \cong A_{(N+3)}(x) \cong \ldots \quad \text { all } \quad x \in E_{1}
$$

hold. This allows to define

$$
\mathscr{D}_{A}=\bigcup_{n=1}^{\infty} D_{n} \quad A: E_{1} \rightarrow L\left(\mathscr{D}_{A}, \mathscr{D}_{A}\right)
$$

$A(x) \uparrow D_{n}=A_{(n)}(x)$ for all $x \in E_{1}$ and all $n=N+1, N+2, \ldots$.

By construction this $A$ is the symmetric linear extension of $A_{(N)}$ which is fixed by $T_{2 N+1}$ and which has the properties (i)-(iv) of Proposition 2.5 and thus defines the minimal extension of $T_{(2 N)}$ which is fixed by $T_{2 N+1}$.

To prove the corresponding statement in Case $\mathrm{B}$ is even simpler because then we know that $A_{(N)}(x), x \in E_{1}$, is already densely defined in $\mathscr{H}_{(N)}$.

Remark. According to Corollary 2.6 and Theorem 2.7 one expects that in Case B of Theorem 2.7 there should be conditions on $T_{(2 N)}$ such that there is exactly one (minimal) extension of $T_{(2 N)}$. This is proven in Part II.

\section{Applications to the Extension Problem for $T_{(2)}=\left\{1, T_{1}, T_{2}\right\}$}

The purpose of this section is threefold:

a) Using the results of Section 1 and 2 we want to characterize all monotone linear continuous extensions of $T_{(2)}$ (Theorem 3.1).

b) We want to substantiate remark b) following Definition 2.4 by showing that the generalized free field associated with a given two-point-functional $T_{2}$ is the (minimal) local extension of a special minimal extension of $T_{(2)}=\left\{1,0, T_{2}\right\}$.

c) We want to discuss why the extension problem for $T_{(2 N)}$ is rather trivial for $N \leqq 1$ but not for $N>1$.

To begin with we formulate

Theorem 3.1. Suppose that the complete tensoralgebra $E$ of $E$ has properties (5).

(a) A given functional $T_{(2)}=\left\{1, T_{1}, T_{2}\right\} \in \underline{E}_{(2)}^{\prime}$ has a m.c.l. extension if and only if

$$
T_{(2)} \uparrow \underline{E}_{+} \cap \underline{E}_{(2)} \geqq 0
$$

holds. 
(b) If $T_{(2)} \in \underline{E}_{(2)}^{\prime}$ satisfies the monotonicity condition (22') the set of all m.c.l. extensions of $T_{(2)}$ can be characterized in terms of their nuclear representations (23) in the following way: Take a nuclear representation $\left(\underline{\tau}_{(1)}\left(\underline{E}_{(1)}\right),\langle\cdot, \cdot\rangle_{2}\right)$ of $T_{(2)}$ according to Theorem 2.1b). Then $T \in E_{+, 1}^{\prime}$ is an extension of $T_{(2)}$ if and only if $a$ matrix representation $m \in \mathfrak{M}_{\infty}^{(2)}\left(E^{\prime}\right)$ of $T$ has the following form:

$$
\left(m_{k j}(x)_{k, j=0,1,2, \ldots}\right)=\left[\begin{array}{cccc}
\tau_{0}^{(1)}(x) & \tau_{1}^{(1)}(x) & \tau_{2}^{(1)}(x) & \ldots \\
\tau_{1}^{(1)}(x) & a_{11}(x) & a_{12}(x) & \ldots \\
\tau_{2}^{(1)}(x) & a_{21}(x) & a_{22}(x) & \ldots \\
\vdots & \vdots & \vdots & \ldots
\end{array}\right]
$$

with some $a \in \mathfrak{M}_{\infty}^{(2)}\left(E^{\prime}\right)$ such that

(i) $\underline{\hat{\underline{t}}}^{(1)}(x)=\left\{\tau_{1}^{(1)}(x), \tau_{2}^{(1)}(x), \ldots\right\}$ is in the domain of all $a\left(x_{1}\right) \ldots a\left(x_{n}\right), x_{j} \in E_{1}, n \in \mathbb{N}$, for all $x \in E_{1}$, where we used the notation $\underline{\tau}_{(1)}\left(x_{0}, x_{1}\right)=x_{0} \underline{\underline{\tau}}^{(0)}+\underline{\tau}^{(1)}\left(x_{1}\right)$ and

$$
\underline{\tau}^{(1)}\left(x_{1}\right)=T_{1}\left(x_{1}\right) \underline{\tau}^{(0)} \oplus \underline{\hat{\tau}}^{(1)}\left(x_{1}\right), \quad \underline{\tau}^{(0)}=\underline{\tau}_{(1)}(\underline{1}) .
$$

(ii) $q_{n}\left(x_{1} \otimes \ldots \otimes x_{n}\right)=\left(\sum_{\nu=1}^{\infty}\left|\left(a\left(x_{1}\right) \ldots a\left(x_{n-1}\right) \hat{\underline{\tau}}^{(1)}\left(x_{n}\right)\right)_{v}\right|^{2}\right)^{1 / 2}$ are continuous seminorms on $E_{1}^{\otimes n}, n=2,3, \ldots$.

Proof. a) Condition (22') is clearly necessary. Suppose now that (22') holds. Then by Theorem $2.1 T_{(2)}$ has a nuclear representation (23):

$$
\begin{aligned}
& \underline{\tau}_{(1)}: \underline{E}_{(1)} \rightarrow l^{2}(\mathbb{C}), \\
& T_{(2)}\left(\underline{x}^{*} \cdot \underline{y}\right)=\left\langle\underline{\tau}_{(1)}(\underline{x}), \underline{\tau}_{(1)}(\underline{y})\right\rangle_{2} \text { for all } \underline{x}, \underline{y} \in \underline{E}_{(1)}=E_{0} \oplus E_{1} .
\end{aligned}
$$

We may identify $\underline{\tau}^{(0)}=\left\{\tau_{0}^{(0)}, \tau_{1}^{(0)}, \tau_{2}^{(0)}, \ldots\right\}$ and $\underline{e}_{0}=\{1,0,0, \ldots\}$. Now any $a(x) \in \mathfrak{M}_{\infty}^{(2)}\left(E^{\prime}\right)$ which satisfies condition (i) and (ii) of Theorem 3.1 (b) gives rise to an element $m \in \mathfrak{M}_{\infty}^{(2)}\left(E^{\prime}\right)$ according to Definition (39). Thus by Theorem 1.2 there is a m.c.l. functional $T_{m, \underline{e}_{0}} \in \underline{E}_{+, 1}^{\prime}$. By construction we have

$$
\begin{aligned}
m(x) \underline{e}_{0} & =\underline{\tau}^{(1)}(x)=T_{1}(x) \underline{e}_{0} \oplus \underline{\hat{\tau}}^{(1)}(x) \\
m\left(x_{1}\right) m\left(x_{2}\right) \underline{e}_{0} & =T_{2}\left(x_{1} \otimes x_{2}\right) \underline{e}_{0} \oplus\left(T_{1}\left(x_{2}\right) \underline{\hat{\tau}}^{(1)}\left(x_{1}\right)+a\left(x_{1}\right) \underline{\hat{\tau}}^{(1)}\left(x_{2}\right)\right)
\end{aligned}
$$

Therefore this matrix state $T_{m, e_{0}}$ defines a m.c.l. extension of $T_{(2)}$. This proves (a) and the sufficiency part of (b).

b) If $T \in \underline{E}_{+, 1}^{\prime}$ is any extension of $T_{(2)}$ then by Theorem $1.2 T$ is a matrix state $T_{m, \underline{z}}$, e.g.

$$
\begin{aligned}
& T_{n}\left(x_{1} \otimes \ldots \otimes x_{n}\right)=\left\langle\underline{z}, m\left(x_{1}\right) \ldots m\left(x_{n}\right) \underline{z}\right\rangle_{2} \quad \text { for all } \quad x_{j} \in E_{1} \text { and } n \in \mathbb{N} . \\
& T^{\uparrow} \underline{\underline{E}}_{(2)}=T_{(2)} \quad \text { implies } \quad T_{1}(x)=\langle\underline{z}, m(x) \underline{z}\rangle_{2}=\tau_{0}^{(1)}(x)
\end{aligned}
$$

and

$$
T_{2}(x \otimes y)=\langle\underline{z}, m(x) m(y) \underline{z}\rangle_{2}=\left\langle m\left(x^{*}\right) \underline{z}, m(y) \underline{z}\right\rangle_{2}=\left\langle\underline{\tau}^{(1)}\left(x^{*}\right), \underline{\tau}^{(1)}(y)\right\rangle_{2},
$$

e.g. eventually after some unitary transformation in $\underline{\tau}_{(1)}\left(\underline{E}_{(1)}\right)$ we may assume $\underline{z}=\underline{e}_{0}$ and $\underline{\tau}^{(1)}(x)=m(x) \underline{z}$. This shows that a matrix representation $m$ of any extension 
$T \in \underline{E}_{+, 1}^{\prime}$ of $T_{(2)}$ has the form specified in (39). Clearly $m \in \mathfrak{M}_{\infty}^{(2)}\left(E^{\prime}\right)$ implies conditions (i) and (ii) of (b).

Remark. (a) Theorem 3.1 shows that a given $T_{(2)} \in E_{(2)}^{\prime}$ which satisfies $\left(22^{\prime}\right)$ has a lot of m.c.l. extensions. This follows easily from the fact that any finite order matrix $a$ satisfies the conditions (i) and (ii) of Theorem 3.1 (b).

(b) One can use formula (20) and Theorem 3.1 to split off explicitly that part of an extension $T \in \underline{E}_{+, 1}^{\prime}$ which is fixed by $T_{(2)}$.

(c) It seems that in the literature only three m.c.l. extensions of $T_{(2)}$ are known:

(i) the trivial one described according to Theorem 3.1 by Equation (39) with $a=0$,

(ii) for $T_{1}=0$ that extension of $T_{(2)}$ which is constructed in QFT via the Fockspace construction over the canonical pre-Hilbertspace $V_{1}=\left(\Phi_{(1)}\left(\underline{E}_{(1)}\right),\langle\cdot, \cdot\rangle_{(1)}\right)$,

(iii) for $T_{1}=0$ a special class of extensions by means of certain scalarproducts on $E_{n}$ using rough estimates [21].

The next topic of this section is to show how the best known examples of m.c.l. extensions of $T_{(2)}$, e.g. case (i) and (ii) of remark (c), fit into the scheme of minimal extensions.

Given $T_{(2)} \in E_{(2)}^{\prime}$ such that $\left(22^{\prime}\right)$ holds there is the canonical pre-Hilbertspace realization $\left(\Phi_{(1)}\left(\underline{E}_{(1)}\right),\langle\cdot, \cdot\rangle_{(1)}\right)=V_{1}$ of $T_{(2)}$. Define $\Phi_{0}=\Phi_{(1)}(\underline{1})$ and $\Phi^{1}(x)=\Phi_{1}(x)$ $-T_{1}(x) \Phi_{0}$ for all $x \in E_{1}$. Then

$$
\Phi_{(1)}\left(x_{0}, x_{1}\right)=\left(x_{0}+T_{1}\left(x_{1}\right)\right) \Phi_{0} \oplus \Phi^{1}\left(x_{1}\right)
$$

and thus $\Phi_{(1)}\left(\underline{E}_{(1)}\right)$ is the orthogonal direct sum

$$
\Phi_{(1)}\left(E_{(1)}\right)=\mathbb{C} \Phi_{0} \oplus \Phi^{1}\left(E_{1}\right) \text {. }
$$

According to this decomposition we define linear operators in $\Phi_{(1)}\left(E_{(1)}\right)$ :

$$
\begin{aligned}
A_{00}(x) \Phi_{0} & =T_{1}(x) \Phi_{0}, \quad A_{10}(x) \Phi_{0}=\Phi^{1}(x) \\
A_{01}(x) \Phi^{1}(y) & =\left\langle\Phi^{1}(x), \Phi^{1}(y)\right\rangle_{1} \Phi_{0} \quad \text { and } \\
A_{(1)}(x) & =\left(\begin{array}{cc}
A_{00}(x) & A_{01}(x) \\
A_{10}(x) & 0
\end{array}\right)
\end{aligned}
$$

for all $x, y \in E_{1}$. This definition yields $A_{(1)}(x) \Phi_{0}=T_{1}(x) \Phi_{0} \oplus \Phi^{1}(x)=\Phi_{1}(x)$ and a simple calculation shows $\left\|A_{(1)}(x)\right\| \leqq\left(T_{2}\left(x^{*} \otimes x\right)+T_{11}\left(x \otimes x^{*}\right)\right)^{1 / 2}$ e.g. $A_{(1)}(x)$ is a bounded linear operator in $V_{1}$ such that $x \mapsto\left\|A_{(1)}(x)\right\|$ is a continuous seminorm on $E_{1}$. The symmetry $T_{1}^{*}=T_{1}$ and $T_{2}^{*}=T_{2}$ of $T_{(2)}$ implies the symmetry $\left(A_{(1)}(x)\right)^{*}$ $=A_{(1)}\left(x^{*}\right)$ of $A_{(1)}$. Therefore a minimal extension of $T_{(2)}$ is welldefined by $\left(x_{j} \in E_{1}, n \in \mathbb{N}\right)$

$$
T_{n}\left(x_{1} \otimes \ldots \otimes x_{n}\right)=\left\langle\Phi_{0}, A_{(1)}\left(x_{1}\right) \ldots A_{(1)}\left(x_{n}\right) \Phi_{0}\right\rangle_{(1)} .
$$

Using Definitions (40) one can express $T_{n}$ explicitly in terms of $T_{1}$ and $T_{2}$. The result is given in the proof of Corollary 1.4.

Knowing this special minimal extension one can easily construct a lot of other minimal and non minimal extensions. Details are contained in Part II, Section 4, Corollary 4.3-4.5. 
Another way of generalizing the minimal extension of $T_{(2)}=\left\{1,0, T_{2}\right\}$ associated with

$$
A_{(1)}(x)=\left(\begin{array}{cc}
0 & A_{01}(x) \\
A_{10}(x) & 0
\end{array}\right)
$$

is obtained by the wellknown Fock-space construction:

We embed $V_{1}$ into the much bigger pre-Hilbertspace $\mathscr{F}$ which is the locally convex direct sum of the tensorproducts of $\Phi^{1}\left(E_{1}\right)$

$$
\mathscr{F}=\bigoplus_{n=0}^{\infty} \mathscr{F}_{n}, \quad \mathscr{F}_{0}=\mathbb{C} \Phi_{0}, \quad \mathscr{F}_{n}=\Phi^{1}\left(E_{1}\right) \otimes \ldots \otimes \Phi^{1}\left(E_{1}\right), \quad n \text {-times },
$$

and construct a symmetric linear extension $A$ of $A_{(1)}$ on $\mathscr{F}$. To this end we define as usual for all $x, x_{j}, y \in E_{1}, n \in \mathbb{N}$

$$
\begin{aligned}
& a^{+}(x) \Phi_{0}=\Phi^{1}(x)=A_{10}(x) \Phi_{0} \\
& a^{+}(x) \Phi^{1}\left(x_{1}\right) \otimes \ldots \otimes \Phi^{1}\left(x_{n}\right)=\Phi^{1}(x) \otimes \Phi^{1}\left(x_{1}\right) \otimes \ldots \otimes \Phi^{1}\left(x_{n}\right) \\
& a^{-}(x) \Phi_{0}=0, \quad a^{-}(x) \Phi^{1}(y)=\left\langle\Phi^{1}(x), \Phi^{1}(y)\right\rangle_{1} \Phi_{0} \\
& a^{-}(x) \Phi^{1}\left(x_{1}\right) \otimes \ldots \otimes \Phi^{1}\left(x_{n}\right)=\left\langle\Phi^{1}\left(x^{*}\right), \Phi^{1}\left(x_{1}\right)\right\rangle_{1} \Phi^{1}\left(x_{2}\right) \otimes \ldots \otimes \Phi^{1}\left(x_{n}\right) \quad n \geqq 2
\end{aligned}
$$

and get bounded linear operators $\left(S_{n}=\right.$ symmetrization operator on $\left.\mathscr{F}_{n}\right)$

$$
\begin{aligned}
& A_{n+1, n}(x)=(n+1)^{1 / 2} S_{n+1} a^{+}(x): \mathscr{\mathscr { F }}_{n} \rightarrow \mathscr{F}_{n+1} \\
& A_{n, n+1}(x)=(n+1)^{1 / 2} a^{-}(x) S_{n+1}: \mathscr{\mathscr { F }}_{n+1} \rightarrow \mathscr{F}_{n}
\end{aligned}
$$

such that

$$
\left\|A_{n+1, n}(x)\right\| \leqq(n+1)^{1 / 2}\left\|\Phi^{1}(x)\right\|_{1}, \quad\left\|A_{n, n+1}(x)\right\| \leqq(n+1)^{1 / 2}\left\|\Phi^{1}(x)\right\|_{1}
$$

and

$$
A_{n, n+1}(x)=\left(A_{n+1, n}\left(x^{*}\right)\right)^{*} \uparrow \widetilde{F}_{n+1} .
$$

This implies that

$$
A(x)=\left[\begin{array}{cccccc}
0 & A_{01}(x) & 0 & 0 & \ldots & \ldots \\
A_{10}(x) & 0 & A_{12}(x) & 0 & 0 & \ldots \\
0 & A_{21}(x) & 0 & A_{23}(x) & 0 & \ldots \\
0 & 0 & A_{32}(x) & 0 & \ldots & \ldots \\
\vdots & \vdots & \vdots & \vdots & \vdots & \vdots
\end{array}\right]
$$

is a welldefined linear operator $\mathscr{\mathscr { F }} \rightarrow \mathscr{F}$ such that $A\left(x^{*}\right)=A(x)^{*} \uparrow \mathscr{F}$ and $A(x) \Phi_{0}$ $=A_{(1)}(x) \Phi_{0}=\Phi_{1}(x)$ hold. $A(x)$ is unbounded but all $A\left(x_{1}\right) \ldots A\left(x_{n}\right)$ are welldefined and $x_{1} \otimes \ldots \otimes x_{n} \mapsto\left\|A\left(x_{1}\right) \ldots A\left(x_{n}\right) \Phi_{0}\right\|_{\mathscr{F}}$ are continuous seminorms on $E_{1}^{\otimes n}$ for all $n \in \mathbb{N}$. The reasons for this simply are the special form of $A(x)$ (the form of a Jacobimatrix) and the continuity properties of $x \mapsto\left\|A_{n m}(x)\right\|$. Thus $A(x)$ defines a m.c.l. extension of $T_{(2)}$ according to

$$
T_{n}\left(x_{1} \otimes \ldots \otimes x_{n}\right)=\left\langle\Phi_{0}, A\left(x_{1}\right) \ldots A\left(x_{n}\right) \Phi_{0}\right\rangle_{\mathscr{F}} .
$$


If $E_{1}=\mathscr{S}\left(\mathbb{R}^{4}\right)$ and if $T_{2}$ satisfies in addition the linear constraints of QFT it is easy to see that the minimal extension of $T_{(2)}=\left\{1,0, T_{2}\right\}$ defined in terms of

$$
A_{(1)}(x)=\left(\begin{array}{cc}
0 & A_{01}(x) \\
A_{10}(x) & 0
\end{array}\right)
$$

satisfies Poincaré-invariance and the spectral condition of QFT but not locality. To construct a m.c.l. extension which is local one could try to construct a minimal extension in terms of

$$
A_{(1)}^{\prime}(x)=\left(\begin{array}{cc}
0 & A_{01}(x) \\
A_{10}(x) & A_{11}(x)
\end{array}\right) \quad\left(A_{11}(x) \text { appropriate }\right)
$$

But this would mean to fix in addition at least a three-point-functional appropriately. In principle it is possible to proceed in this way (Part II, Section 3). But such a kind of extension is not applicable if one wants to have the usual association between particles and fields.

The extension of $T_{(2)}$ that we get by restricting the above $A: E_{1} \rightarrow L(\mathscr{F}, \mathscr{F})$ to $\mathscr{D}_{A}=\bigoplus_{n=0}^{\infty} S_{n} \mathscr{F}_{n}$ to get $A: E_{1} \rightarrow L\left(\mathscr{D}_{A}, \mathscr{D}_{A}\right)$ is the well-known Fock-space extension of $T_{(2)}=\left\{1,0, T_{2}\right\}$; it is a local extension of the minimal extension of $T_{(2)}$ which is fixed by $T_{3}=0$. This Fock-space extension of $T_{(2)}$ is minimal in the sense that only the given two-point-functional $T_{2}$ and some combinatorial objects which take care of locality are involved.

Another way of constructing the Fock-space extension is to use in Theorem 3.1 a special matrix $m \in D\left(E^{\prime}\right)$ :

$$
m(x)=\sum_{j=0}^{\infty}\left\{\tau_{j}^{(1)}(x) a^{(j)}+\tau_{j}^{(1)}(x) a^{(j)+}\right\}
$$

$\tau_{j}^{(1)}(x)$ as in Theorem 3.1; $\left\{a^{(j)}, a^{(j)+}\right\}$ a suitable matrix realization of the canonical commutation relations.

As a last point of this section we indicate the main differences of the extension problem of $T_{(2 N)}$ for $N \leqq 1$ and $N>1$ :

$(\alpha)$ For $N \leqq 1$ the obviously necessary continuity conditions on $T_{(2 N)}$ of type (29) are implied by monotonicity $T_{(2 N)} \geqq 0$ but not for $N>1$. For $N>1$ these continuity conditions are independent restrictions.

$(\beta)$ For $N \leqq 1$ the linear function $A_{(1)}: E_{1} \rightarrow L\left(V_{0}, V_{1}\right)$ which yields $T_{(2)}$ in the canonical pre-Hilbertspace realization is always a bounded operator valued function [realization (40)]. Therefore a lot of minimal and thus also non minimal extensions always exist in this case. For $N>1$ this is not necessarily true (see examples of Part II).

$(\gamma)$ The possibility (C) of Theorem 1.4 of Part II (e.g. there is no extension at all) is excluded in a trivial way for $N \leqq 1$.

\section{The Problem of Monotone Extensions for $E \cong \mathbb{C}$ (Moment Problem)}

This section is intended

a) to illustrate how the idea of minimal extensions works in the simplest case, e.g. in the case where $E_{1}$ is one-dimensional: $E_{1}=\mathbb{C} e_{1}$. 
b) To show how one can construct all m.c.l. extensions in this case knowing all minimal extensions and

c) to determine the structure of elements in $\mathfrak{M}_{\infty}^{(2)}(\mathbb{C})$.

Of course it would be very helpful to know the structure of elements in $\mathfrak{M}_{\infty}^{(2)}\left(E^{\prime}\right)$ for general $E$. This analysis gives a hint what one can expect.

For $E_{1}=\mathbb{C} e_{1}$ we have $E_{n}=\mathbb{C} e_{n}, e_{n}=e_{1} \otimes \ldots \otimes e_{1}, n$-times and the topology of the locally convex direct sum

$$
E=\bigoplus_{n=0}^{\infty} E_{n}
$$

is defined by all sequences $\left\{c_{n} \geqq 0\right\}_{n \in \mathbb{N}}$ according to the definition of continuous seminorms

$$
q_{c_{n}}(\underline{z})=\sum_{n=0}^{\infty} c_{n}\left|z_{n}\right|
$$

With the definition of the product $(\underline{\zeta} \cdot \underline{z})_{n}=\sum_{\nu+\mu=n} \zeta_{\nu} z_{\mu}$ and the involution

$$
(\underline{z})^{*}=\left(\sum_{n=0}^{\infty} z_{n} e_{n}\right)^{*}=\sum_{n=0}^{\infty} \bar{z}_{n} e_{n}
$$

$\underline{E}$ becomes a $*$-algebra with unit. Clearly all $E_{n}$ are barreled and nuclear; thus our general assumptions on $\underline{E}$ are fulfilled, e.g. $\underline{E}$ is a barreled nuclear locally convex *-algebra with unit.

Clearly the normalized m.c.l. functionals $t=\left\{1, t_{1}, t_{2}, \ldots\right\} \in \underline{E}_{+, 1}^{\prime}$ on $\underline{E}$ and the sequences $\underline{c}=\left\{1, c_{1}, c_{2}, \ldots\right\}$ of positive type, e.g. those sequences $\underline{c}$ such that for all $N \in \mathbb{N}$ and all $\left\{z_{0}, z_{1}, \ldots, z_{N}\right\} \in \mathbb{C}^{N+1}$ we have

$$
\sum_{n, m=0}^{N} \bar{z}_{n} c_{n+m} z_{m} \geqq 0
$$

are in a one to one correspondence according to $c_{n}=t_{n}\left(e_{n}\right)$ for all $n \in \mathbb{N}$. Therefore in the case $E_{1} \cong \mathbb{C}$ Theorem 1.2 is a characterization of sequences of positive type:

Corollary 4.1. A sequence $\underline{c}=\left\{1, c_{1}, c_{2}, \ldots\right\}$ is of positive type if and only if there is an element $m$ in $\mathfrak{M}_{\infty}^{(2)}(\mathbb{C})$ such that

$$
c_{1}=m_{00}
$$

and

$$
c_{n+1}=\sum_{j_{1}, \ldots, j_{n}=0}^{\infty} m_{0 j_{1}} m_{j_{1} j_{2}} \ldots m_{j_{n} 0} .
$$

But this is nothing else than the well-known [23] result:

Corollary 4.2. A sequence $\underline{c}=\left\{1, c_{1}, c_{2}, \ldots\right\}$ is of positive type if and only if there is a symmetric operator $A$ in some separable Hilbertspace $\mathscr{H}$ which contains a cyclic unit-vector $e_{0}$ for $\left\{A^{n} \mid n=0,1, \ldots\right\}$ such that

$$
c_{n}=\left(e_{0}, A^{n} e_{0}\right) \quad n=0,1, \ldots .
$$

This in turn can be used to show (see Appendix): 
Proposition 4.3. Each $m \in \mathfrak{M}_{\infty}^{(2)}(\mathbb{C})$ is unitarily equivalent (in the sense of von Neumann) to an element $a \in \mathfrak{M}_{\infty}^{(2)}(\mathbb{C})$ in standard form

$$
a=\left[\begin{array}{cccccc}
a_{00} & a_{01} & 0 & 0 & 0 & \ldots \\
a_{10} & a_{11} & a_{12} & 0 & 0 & \ldots \\
0 & a_{21} & a_{22} & a_{23} & 0 & \ldots \\
0 & 0 & a_{32} & a_{33} & a_{34} & \ldots \\
: & : & . & & & \ldots
\end{array}\right]
$$

Knowing the standard form of elements in $\mathfrak{M}_{\infty}^{(2)}(\mathbb{C})$ we can determine all m.c.l. extensions of a given $t_{(2 N)}=\left\{1, c_{1}, \ldots, c_{2 N}\right\} \in \underline{E}_{(2 N)}^{\prime} \cong \mathbb{R}^{2 N+1}$ such that

$$
\begin{aligned}
& t_{(2 N)} \backslash \underline{E}_{+} \cap \underline{E}_{(2 N)} \geqq 0, \quad \text { e.g. } \\
& \sum_{n, m=0}^{N} \bar{z}_{n} c_{n+m} z_{m} \geqq 0, \quad\left\{z_{0}, \ldots, z_{N}\right\} \in \mathbb{C}^{N+1}
\end{aligned}
$$

To solve this problem we have to find all real numbers $c_{n}, n=2 N+1,2 N+2, \ldots$ such that $\underline{c}=\left\{1, c_{1}, c_{2}, \ldots\right\}$ is a sequence of positive type in the sense of relation (42). According to Theorem 2.1 we know that by assumption (44) there is a continuous linear map $\Phi_{(N)}$ from $\underline{E}_{(N)}$ onto some pre-Hilbertspace $V_{N}=\left(\Phi_{(N)}\left(E_{(N)}\right),\langle\cdot, \cdot\rangle_{(N)}\right)$ such that

$$
\begin{aligned}
& \begin{aligned}
t_{(2 N)}\left(\underline{z}^{*} \cdot \zeta\right) & =\left\langle\Phi_{(N)}(\underline{z}), \Phi_{(N)}(\zeta)\right\rangle_{(N)} \\
& =\sum_{n, m=0}^{N} \bar{z}_{n} \zeta_{m}\left\langle\Phi_{n}\left(e_{n}\right), \Phi_{m}\left(e_{m}\right)\right\rangle_{(N)}
\end{aligned} \\
& \text { e.g. } c_{n+m}=\left\langle\Phi_{n}\left(e_{n}\right), \Phi_{m}\left(e_{m}\right)\right\rangle_{(N)} \text { for all } n, m \in\{0,1, \ldots, N\} \\
& V_{N} \text { is spanned by }\left\{\Phi_{0}\left(e_{0}\right), \ldots, \Phi_{N}\left(e_{N}\right)\right\} .
\end{aligned}
$$

To apply the idea of minimal extensions we have to construct a symmetric operator $A_{(N)}$ in $V_{N}$ such that

$$
\Phi_{n}\left(e_{n}\right)=A_{(N)}^{n} \Phi_{0}\left(e_{0}\right) \quad n=0,1, \ldots, N
$$

holds, and then the definition

$$
c_{n}=\left\langle\Phi_{0}\left(e_{0}\right), A_{(N)}^{n} \Phi_{0}\left(e_{0}\right)\right\rangle_{(N)} \quad n=2 N+1, \quad 2 N+2, \ldots
$$

yields a minimal extension. The following proposition says that this always works.

Proposition 4.4. Given a sequence $t_{(2 N)}=\left\{1, c_{1}, \ldots, c_{2 N}\right\}$ of real numbers which satisfies condition (44) there exist minimal extensions of $t_{(2 N)}$ which are constructed according to Equation (46).

a) If $V_{N}$ is spanned by $\left\{\Phi_{0}\left(e_{0}\right), \ldots, \Phi_{M}\left(e_{M}\right)\right\}, M<N$ (notation of relation (45)) there is exactly one m.c.l. extension and this is a minimal extension of $t_{(2 N)}$.

b) If $\operatorname{dim}\left(V_{N}\right)=N+1$ the cardinality of the set of all minimal extensions of $t_{(2 \mathrm{~N})}$ equals the cardinality of $\mathbb{R}$. The most general m.c.l. extension of $t_{(2 \mathrm{~N})}$ is obtained 
according to Corollary 4.1 by an element $m \in \mathfrak{M}_{\infty}^{(2)}(\mathbb{C})$ such that

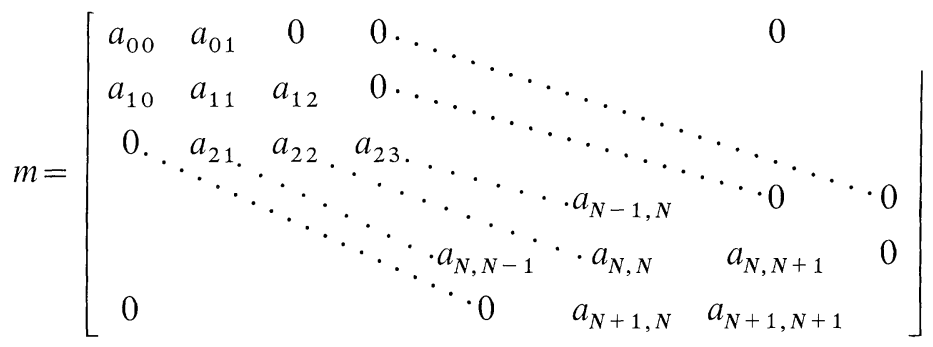

where $a_{n m}=\bar{a}_{m n}, 0 \leqq n \leqq N, 0 \leqq m \leqq N-1$ is fixed by $t_{(2 N)}$ and $a_{n m}=\bar{a}_{m n}, n, m \geqq N$ is arbitrary.

We prove Proposition 4.4 in the Appendix.

Remark. (a) In Part II we derive explicit conditions on $t_{(2 N)}$ (for general $E$ ) which allow to distinguish case a) and b) of Proposition 4.4.

(b) Another version of the existence and uniqueness part of Proposition 4.4 is for instance contained in [22,23].

(c) Notice the simple connection of the extension problem for $E_{1} \cong \mathbb{C}$ with the extension problem in the case of a general HLCTVS $E_{1}$ : Given $T_{(2 N)} \in E_{(2 N)}^{\prime}$, $T_{(2 N)} \geqq 0$, and $x^{*}=x \in E_{1}$ define

$$
c_{n}=T_{n}\left(x^{\otimes n}\right), \quad n=1, \ldots, 2 N
$$

then $t_{(2 N)}=\left\{1, c_{1}, \ldots, c_{2 N}\right\}$ is a sequence of real numbers which satisfies relation (44). Thus for each fixed $x^{*}=x \in E_{1}$ we know all the solutions of the extension problems. Therefore to solve the extension problem for $T_{(2 N)}$ means to find conditions which imply that these various extensions fit together in a proper way.

\section{Appendix}

This appendix contains the proofs of the statements of Section 4.

Proof of Proposition 4.3. According to Corollary 4.1 and 4.2 an element $m$ in $\mathfrak{M}_{\infty}^{(2)}(\mathbb{C})$ can be thought of as the matrix representation of a symmetric operator in some separable Hilbertspace $\mathscr{H}$ with respect to an orthonormal basis $\left\{e_{n ; n=0,1,2, \ldots}\right\}$ of $\mathscr{H}$ in the domain $D(A)$ of this operator $A: m_{i j}=\left(e_{i}, A e_{j}\right)$ $i, j=0,1, \ldots$ such that $e_{0}$ is cyclic for $\left\{A^{n} \mid n=0,1, \ldots\right\}$. Then we know that $\left\{A^{n} e_{0} \mid n=0,1,2, \ldots\right\}$ contains a basis of $\mathscr{H}$ contained in $D(A)$. By orthonormalization of this basis we get an orthonormal basis $\left\{f_{0}=e_{0}, f_{1}, f_{2}, \ldots\right\}$ of $\mathscr{H}$ in $D(A)$ :

$$
\begin{aligned}
& f_{n}=\sum_{j=0}^{n} \alpha_{n j} A^{j} e_{0} \quad \sum_{j=0}^{n} \bar{\alpha}_{n j} \alpha_{m n}=\delta_{n m} \\
& A^{n} e_{0}=\sum_{j=0}^{n} \gamma_{n j} f_{j} \quad \text { for all } n \in \mathbb{N} .
\end{aligned}
$$


If we calculate now the matrix representation of $A$ with respect to the basis $\left\{f_{0}, f_{1}, \ldots\right\} \subset D(A)$ we obtain

$$
\left(f_{n}, A f_{m}\right)=\sum_{i=0}^{m} \alpha_{m i} \sum_{j=0}^{i+1} \gamma_{i+1, j} \delta_{n j}
$$

and therefore $\left(f_{n}, A f_{m}\right)=0$ whenever $n \geqq m+2$ and thus by symmetry of $A$

$$
\left(f_{n}, A f_{m}\right)=0 \quad \text { if } \quad|n-m| \geqq 2 .
$$

This shows that $\left(m_{i j}\right)$ and $\left(a_{i j}=\left(f_{i}, A f_{j}\right)\right)$ are unitarily equivalent in the sense of von Neumann and that $a=\left(a_{i j}\right)$ has standard form.

Proof of Proposition 4.4. If $t_{(2 N)}$ satisfies (44) it has a canonical pre-Hilbertspace realization $V_{N}$ such that the relations (45) hold. Let us denote $f_{n}=\Phi_{n}\left(e_{n}\right)$, $n=0, \ldots, N$.

a) If $V_{N}$ is spanned by $\left\{f_{0}, \ldots, f_{M}\right\}$ we obtain

$$
f_{n}=\sum_{j=0}^{M} \beta_{n j} f_{j} \quad M+1 \leqq n \leqq N .
$$

Therefore the definition of $A_{(N)}$ is already fixed by condition (46a):

$$
\begin{aligned}
& A_{(N)} f_{n-1}=f_{n} \quad n=1, \ldots, M \\
& A_{(N)} f_{M}=f_{M+1}=\sum_{j=0}^{M} \beta_{M+1, j} f_{j} .
\end{aligned}
$$

The relations (45b) imply that with this definition $A_{(N)}$ is a well-defined symmetric operator in $V_{N}$. These relations also imply that the Equations (46a) are also fulfilled for $n=M+1, \ldots, N$ :

If $M=N-1$ this is the case according to definition (A.4). If $M<N-1$ we calculate for arbitrary

$$
g=\sum_{j=0}^{M} \beta_{j} f_{j} \in V_{M}=\operatorname{lin}\left\{f_{0}, \ldots, f_{M}\right\}
$$

using (45b) and the definition of $A_{(N)}\left\langle g, f_{M+2}\right\rangle_{(N)}=\left\langle g, A_{(N)}^{2} f_{M}\right\rangle_{(N)}$ thus $A_{(N)}^{2} f_{M}=f_{M+2}$. If $M<N-2$ a finite number of similar steps yields the result. Therefore there is a minimal extension of $t_{(2 N)}$ in case a) of Proposition 4.4.

Suppose now that $t=\left\{1, c_{1}, c_{2}, \ldots\right\}$ is any m.c.l. extension of $t_{(2 N)}$. It has a canonical pre-Hilbertspace realization $V_{t}=\left(\Phi_{t}(E),\langle\cdot, \cdot\rangle_{t}\right)$ and we may assume $\Phi_{t} \backslash \underline{E}_{(N)}=\Phi_{(N)}$. Otherwise we had to do a unitary transformation in $V_{N}$. Then we have vectors $f_{n}=\Phi_{n}\left(e_{n}\right), n=0,1,2, \ldots$ in $V_{t}$ and a symmetric operator $A: V_{t} \rightarrow V_{t}$ such that $f_{n+1}=A f_{n} n=0,1, \ldots$ holds. Together with (A.3) this implies $f_{n} \in V_{M}$ for all $n \geqq M+1$, e.g. $V_{t}=V_{M}=V_{N}$; that is $t$ is a minimal extension of $t_{(2 N)}$. Then it is clear that $A$ is fixed by its action on $V_{M}$, e.g. by $A_{(N)}$; therefore there is exactly one m.c.l. extension of $t_{(2 N)}$.

b) If $V_{N}$ has maximal dimension $N+1$ the condition (46a) defines the operator $A_{(N)}$ in $V_{N}$ we are looking for only on the proper subspace $V_{N-1}=\operatorname{lin}\left\{f_{0}, \ldots, f_{N-1}\right\}$. 
If we orthogonalize the basis $\left\{f_{0}, \ldots, f_{N}\right\}$ of $V_{N}$ we get an orthonormal basis $\left\{g_{0}=f_{0}, g_{1}, \ldots, g_{N}\right\}$ such that

$$
g_{n}=\sum_{j=0}^{n} \alpha_{n j} f_{j}, \quad f_{n}=\sum_{j=0}^{n} \gamma_{n j} g_{j} .
$$

A calculation just as in the proof of Proposition 4.3 shows that all matrix elements of $A_{(N)}$ with respect to the basis $\left\{g_{0}, \ldots, g_{N}\right\}$ are fixed with the exception of $\left\langle g_{N}, A_{(N)} g_{N}\right\rangle_{(N)}$ :

$$
\begin{aligned}
& a_{n m}=\left\langle g_{n}, A_{(N)} g_{m}\right\rangle_{(N)}=\sum_{i=0}^{m} \alpha_{m i} \sum_{j=0}^{i+1} \gamma_{i+1, j} \delta_{n j} \\
& m=0,1, \ldots, N-1 ; \quad n=0,1, \ldots, N .
\end{aligned}
$$

Each choice $\left\langle g_{N}, A_{(N)} g_{N}\right\rangle_{(N)}=a_{N N}$ is possible and fixes $c_{2 N+1}=\left\langle f_{N}, A_{(N)} f_{N}\right\rangle_{(N)}$ and is in turn fixed by choice of $c_{2 N+1}$. Thus each choice of $c_{2 N+1} \in \mathbb{R}$ defines a symmetric operator $A_{(N)}$ in $V_{N}$ such that (46a) holds. This proves the first part of Proposition 4.4b).

Each infinite matrix of the form described in Proposition 4.4b) where the $a_{n m}$ $n=0, \ldots, N ; m=0, \ldots, N-1$ are calculated as in (A.6) yields according to Proposition 4.3 and Corollary 4.1 a m.c.l. extension of $t_{(2 N)}$. Again by Proposition 4.3 and Corollary 4.1 we see that any extension of $t_{(2 N)}$ can be realized in this way.

\section{References}

1. Bogulubov, N.N., Logunov, A. A., Todorov, I. T.: Introduction to axiomatic quantum field theory. London: Benjamin 1975

2. Floret, K., Wloka,J.: Einführung in die Theorie der lokalkonvexen Räume. Berlin-Heidelberg-New York: Springer 1968

3. Jameson,G.: Ordered linear spaces. Berlin-Heidelberg-New York: Springer 1970

4. Jost, R.: The general theory of quantized fields. Providence, R.I. : American Mathematical Society 1965

5. Pietsch, A.: Nukleare lokalkonvexe Räume. Berlin: Akademie-Verlag 1965

6. Robertson, A.P., Robertson,W.J.: Topologische Vektorräume. Mannheim: Bibliographisches Institut 1967

7. Schaefer,H.H.: Topological vector spaces. 3. Printing. Berlin-Göttingen-Heidelberg-New York: Springer 1964

8. Streater, R.F., Wightman, A.S.: PCT, spin and statistics and all that. New York: W.A. Benjamin 1964

9. Treves, F.: Topological vector spaces, distributions and kernels. New York: Academic Press 1967

10. Wloka,J.: Grundräume und verallgemeinerte Funktionen. Berlin-Heidelberg-New York: Springer 1968

11. Baumann, K.: When is a field theory a generalized free field? Commun. math. Phys. 43, 221-223 (1975)

12. Borchers,H.J.: On the structure of the algebra of field operators. Nuovo Cimento 24, 214 (1962)

13. Borchers,H.J.: Algebraic aspects of Wightman field theory, in statistical mechanics and field theory (ed. R. N. Sen, C. Weil) (Haifa Summer School 1971). Jerusalem: Israel University Press 1972

14. Borchers, H.J.: Einführung in die Quantenfeldtheorie I+II. Vorlesungen an der Universität Göttingen 1966/67

15. Glaser,V.: The positivity condition in momentum space. In: Problems of theoretical physics, pp. 68-89. Moscow: Nauka 1969

16. Lassner, G.: Rept. Math. Phys. 3, 279-293 (1972)

17. Lassner, G., Uhlmann, A. : On positive functionals. Commun. math. Phys. 7, 152-159 (1968) 
18. Neumann, J.von: Zur Theorie der unbeschränkten Matrizen. In: Collected works, Vol. II, pp. 144-172. London: Pergamon Press 1961

19. Wyss, W.: The field algebra and its positive linear functionals. Commun. math. Phys. 27, 223-234 (1972)

20. Bros,J., Lasalle, M.: Analyticity properties and many-particle structure in general quantum field theorie I, II, III. Commun. math. Phys. 36, 185-225 (1974); 43, 279-309 (1975); 54, 33-62 (1977)

21. Yngvason,J.: On the algebra of test functions for field operators. Decomposition of linear functionals into positive one's. Commun. math. Phys. 34, 315-333 (1973)

22. von Mises, R. : Mathematical theory of probability and statistics. New York: Academic Press 1964

23. Shohat,J. A., Tamarkin,J.D.: The problem of moments. Providence: Rhode Island 1943

Communicated by R. Haag

Received August 10, 1977 